\title{
Teste de Propriedades em Torneios
}

\author{
Henrique Stagni \\ DISSERTAÇÃO APRESENTADA \\ AO \\ Instituto de Matemática e Estatística \\ DA \\ Universidade DE SÃo PAUlo \\ PARA \\ OBTENÇÃO DO TÍTULO \\ DE \\ Mestre em CiÊnCIAS \\ Programa: Ciência da Computação \\ Orientador: Prof. Dr. Yoshiharu Kohayakawa
}

Durante o desenvolvimento deste trabalho o autor recebeu auxílio financeiro do CNPq

São Paulo, abril de 2015 


\section{Teste de Propriedades em Torneios}

Esta versão da dissertação contém as correções e alterações sugeridas pela Comissão Julgadora durante a defesa da versão original do trabalho, realizada em 26/01/2015. Uma cópia da versão original está disponível no

Instituto de Matemática e Estatística da Universidade de São Paulo.

Comissão Julgadora:

- Prof. Dr. Yoshiharu Kohayakawa (orientador) - IME-USP

- Prof. Dr. Carlos Hoppen - UFRGS

- Prof. Dr. Daniel Morgato Martin - UFABC 


\section{Resumo}

STAGNI, Henrique. Teste de Propriedades em Torneios. Dissertação - Instituto de Matemática e Estatística, Universidade de São Paulo, São Paulo, 2014.

Teste de propriedades em grafos consiste no estudo de algoritmos aleatórios sublineares que determinam se um grafo $G$ de entrada com $n$ vértices satisfaz uma dada propriedade ou se é necessário adicionár ou remover mais do que $\epsilon\left(\begin{array}{c}n \\ 2\end{array}\right)$ arestas para fazer $G$ satisfazê-la, para algum parâmetro de erro fixo $\epsilon$. Uma propriedade de grafos $\mathcal{P}$ é dita testável se, para todo $\epsilon>0$, existe um tal algoritmo para $\mathcal{P}$ cujo tempo de execução é independente de $n$. Um dos resultados de maior importância nesta área, provado por Alon e Shapira, afirma que que toda propriedade hereditária de grafos é testável.

Neste trabalho, apresentamos resultados análogos para torneios - grafos completos nos quais são dadas orientações para cada aresta.

Palavras-chave: teste de propriedades, torneios, lema de regularidade. 


\section{Abstract}

STAGNI, Henrique. Property Testing in Tournaments Thesis - Instituto de Matemática e Estatística, Universidade de São Paulo, São Paulo, 2014.

Graph property testing is the study of randomized sublinear algorithms which decide if an input graph $G$ with $n$ vertices satisfies a given property or if it is necessary to add or remove more than $\epsilon\left(\begin{array}{l}n \\ 2\end{array}\right)$ edges to make $G$ satisfy it, for some fixed error parameter $\epsilon$. A graph property $\mathcal{P}$ is called testable if, for every $\epsilon>0$, there is such an algorithm for $\mathcal{P}$ whose run time is independent of $n$. One of the most important results in this area is due to Alon and Shapira, who showed that every hereditary graph property is testable.

In this work, we show analogous results for tournaments - complete graphs in which every edge is given an orientation.

Keywords: property testing, tournaments, regularity lemma. 


\section{Sumário}

Notação $\quad$ vii

1 Introdução 1

2 Conceitos Iniciais $\quad 3$

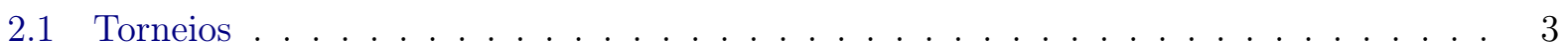

2.2 Teste de Propriedade . . . . . . . . . . . . . . . . . 5

2.3 Exemplo: transitividade é uma propriedade testável . . . . . . . . . . . . . . . . . 9

3 Inevitabilidade e Abundância de subtorneios $\quad 11$

3.1 Regularidade em torneios . . . . . . . . . . . . . . . . . . . . 12

3.1 .1 Definições iniciais . . . . . . . . . . . . . . . . . . . . . . . . . 12

3.1 .2 Lema de regularidade . . . . . . . . . . . . . . . . . . . . . 14

3.1 .3 Considerações sobre o Lema . . . . . . . . . . . . . . . . . . . . . . . . . . 19

3.2 Imersão de torneios em pares regulares . . . . . . . . . . . . . . . . . . . . . 19

4 Testando subtorneios $\quad 23$

4.1 Abundância de um subtorneio é fechada por homomorfismo . . . . . . . . . . . . . . 24

4.2 Lema forte da regularidade . . . . . . . . . . . . . . . . . . 25

4.3 Demonstração do Lema da Remoção para Torneios . . . . . . . . . . . . . . . . . 28

4.4 Subtorneios facilmente testáveis . . . . . . . . . . . . . . . . . . . . . . . 29

5 Caracterização das propriedades testáveis com erro-unilateral 31

5.1 Testando famílias infinitas de subtorneios . . . . . . . . . . . . . 31

5.2 Propriedades semi-hereditárias . . . . . . . . . . . . . . . . . 33

6 Caracterização de propriedades testáveis 35

6.1 Exemplo de propriedade não testável . . . . . . . . . . . . . . . . . . 35

7 Considerações finais $\quad 39$

7.1 Subtorneios facilmente testáveis . . . . . . . . . . . . . . . . . . . 40

7.2 Resultados adicionais para torneios . . . . . . . . . . . . . . . . 41

A Desigualdade de Chebyshev e Cotas de Chernoff 43 


\section{Notação}

- Denotamos o conjunto $\{1,2, \ldots, n\}$ por $[n]$.

- Escrevemos $f: A \longmapsto B$ ao definir uma função $f \in B^{A}$ injetora.

- Dados $d, \eta, \epsilon \in \mathbb{R}$, escrevemos $d=\eta \pm \epsilon$ para indicar que $\eta-\epsilon \leq d \leq \eta+\epsilon$.

- Definimos a função TOWER $: \mathbb{N} \rightarrow \mathbb{N}$ dada por: $\operatorname{TOWER}(0)=1$ e $\operatorname{TOWER}(k)=2^{\operatorname{TOWER}(k-1)}$, para todo $k>0$.

- Definimos a função WOW $: \mathbb{N} \rightarrow \mathbb{N}$ dada por WOW $(0)=1$ e WOW $(k)=\operatorname{TOWER}(\operatorname{WOW}(k-1))$, para todo $k>0$.

- Para uma função $f: \mathbb{R} \rightarrow \mathbb{R}$, definimos $f^{(1)}(x)=f(x)$ e $f^{(n)}(x)=f\left(f^{(n-1)}(x)\right)$ para todo $n>1$.

- Escrevemos $f(x)=\operatorname{poli}(x)$ para indicar que a função $f$ pode ser limitada por polinômio em $x$.

- Escrevemos $\delta(\epsilon)=\operatorname{poli}_{<}(\epsilon)$ para denotar que a função $\epsilon^{-1} \mapsto(\delta(\epsilon))^{-1}$ pode ser limitada por um polinômio em $\epsilon^{-1}$.

- Ao fazermos referências, colocamos um subíndice gr para indicar que o resultado referenciado não é sobre torneios, tratando-se na verdade de uma versão análoga (do resultado em questão) para o caso de grafos (ou digrafos). 
viii NOTAÇÃO 


\section{Capítulo 1}

\section{Introdução}

Teste de propriedades consiste no estudo de algoritmos de decisão capazes de distinguir (com alta probabilidade) se um objeto de entrada possui uma certa propriedade ou se está longe de satisfazê-la (de acordo com alguma métrica no espaço dos objetos considerados) [20]. Tais algoritmos, denominados testadores, devem consumir tempo sublinear no tamanho da entrada, isto é, apenas uma pequena parte dos objetos considerados pode ser inspecionada.

O estudo desse tipo de algoritmo é motivado por situações em que o tamanho dos objetos considerados é tão grande que não é desejável, ou até mesmo possível, sequer ler toda a entrada. Ainda assim, gostaríamos de determinar, mesmo que de forma aproximada, se tais objetos satisfazem uma certa propriedade. Testadores também podem ser usados como um pré-processamento que rapidamente descarta objetos que estão longe de satisfazer a propriedade de interesse, permitindo que um algoritmo de decisão exata seja aplicado apenas às demais entradas.

Testadores de propriedades foram inicialmente considerados no contexto de verificação de corretude de programas. Blum, Luby e Rubinfeld [12] mostraram ser possível testar se a função computada por um dado programa é linear após examinar os valores que ela assume em apenas alguns pontos amostrados do domínio. Rubinfeld e Sudan [29] formalizaram o conceito de teste de propriedades pela primeira vez (ver [16]) ao estudar outras propriedades algébricas de funções, além de linearidade, que também podem ser testadas.

Em [21], Goldreich, Goldwasser e Ron fazem uma abordagem mais genérica que considera teste de propriedades como um modelo computacional aplicável a diversos tipos de objetos. Também nesse trabalho, testadores de propriedades de grafos são considerados pela primeira vez. Ao estudar este tipo de algoritmo, é necessário especificar uma medida de distância entre grafos. No dito modelo denso (um dos dois modelos mais estudados, sendo o outro restrito a grafos com grau máximo limitado [19]), a distância entre dois grafos é dada pela fração de arestas que devem ser adicionadas ou removidas do primeiro para torná-lo isomorfo ao segundo. Além disso, o acesso ao grafo de entrada deve ser feito por meio de consultas que determinam se um par de vértices induz ou não uma aresta. Dadas tais restrições, um parâmetro de erro $0<\epsilon<1$ é fixado, e testadores para uma propriedade $\mathcal{P}$ devem (com alta probabilidade) aceitar grafos que satisfazem $\mathcal{P}$ e rejeitar grafos $\epsilon$-longe de $\mathcal{P}$, isto é, grafos cuja distância àqueles que satisfazem $\mathcal{P}$ é maior que $\epsilon$.

Ao se considerar teste de propriedades em grafos, boa parte da atenção é voltada a algoritmos que fazem um número constante de consultas, isto é, independente do tamanho da entrada nesse caso, a eficiência de um testador é medida pela dependência do número de consultas com o parâmetro de erro $\epsilon$. Mesmo com essa restrição, Goldreich, Goldwasser e Ron [21] mostraram que 
uma certa classe de propriedades - incluindo algumas cujo problema de decisão exato é $\mathcal{N} \mathcal{P}$-difícil, tais como $k$-colorabilidade - são testáveis. De forma ainda mais surpreendente, Alon e Shapira [9] mostraram que todas as propriedades hereditárias de grafos são testáveis em tempo constante. Alon, Fischer, Newman e Shapira [5] forneceram uma caracterização completa das propriedades de grafos testáveis (com número constante de consultas), envolvendo conceitos sobre regularidade em grafos. Uma outra abordagem de teste de propriedades, que faz uso de objetos limite não será exposta neste trabalho. Recomendamos ao leitor interessado em tal abordagem consultar a monografia de Lovász [26, Capítulo 15].

Fischer [17] estendeu alguns dos resultados conhecidos à época para outras estruturas combinatórias, como torneios - grafos completos em que cada aresta recebe uma orientação. O foco deste trabalho consiste no estudo de teste de propriedades em tal estrutura.

No próximo capítulo, fixamos a notação sobre torneios e introduzimos os conceitos básicos a respeito de teste de propriedades. No capítulo 3 apresentamos ferramentas, tais como o Lema de Regularidade de Szemerédi, que serão usadas no capítulo 4 para mostrar que, para qualquer subtorneio $T_{h}$ fixo, a propriedade "ser livre de cópias de $T_{h}$ " é testável. Também mostramos que para uma certa classe de subtorneios $T_{h}$, fechada sobre uma operação de blow-up de vértices, tal propriedade pode ser facilmente testável (isto é, testável com um número de consultas apenas polinomial em relação ao parâmetro de erro $\epsilon$ ), o que evidencia em relação a teste de propriedades de grafos - apenas um conjunto finito de subgrafos $H$ é tal que a propriedade de grafos "ser livre de cópias de $H$ " é facilmente testável.

No capítulo 5 generalizamos resultados obtidos no capítulo anterior para mostrar que qualquer propriedade hereditária de torneios é testável. Também mostramos que essas são, essencialmente, as únicas propriedades testáveis sobre uma certa restrição usual (a saber, quando exigimos que torneios que satisfazem a propriedade de interesse sejam aceitos com probabilidade 1) e mostramos, no capítulo 6, um exemplo de uma propriedade de torneio que não pode ser testada. No capítulo 7 , retomamos os resultados apresentados e os comparamos com os resultados análogos existentes para grafos (e digrafos). 


\section{Capítulo 2}

\section{Conceitos Iniciais}

Neste capítulo, damos a definição e alguma propriedades básicas de torneios que serão necessárias nos capítulos seguintes. Também introduzimos os conceitos básicos a respeito de teste de propriedades e damos um exemplo de uma propriedade testável de torneios.

\subsection{Torneios}

Definição 2.1 (digrafo). Um digrafo é um par $D=(V, A)$, onde $V$ é um conjunto finito e $A \subseteq$ $(V \times V) \backslash\{(v, v): v \in V\}$ é um conjunto de pares ordenados de elementos distintos de $V$.

Os elementos de $V$ são os vértices e os de $A$ são os arcos do digrafo $D$. Dado um digrafo $D$, também denotamos esses conjuntos por $V(D)$ e $A(D)$, respectivamente. Um arco $(u, v)$ também será denotado por $\overrightarrow{u v}$. Se um arco $\overrightarrow{u v} \in A$, dizemos que a orientação desse arco é de $u$ para $v$. Nesse caso, também dizemos que $u$ domina $v$ e denotamos essa relação por $u \stackrel{D}{\rightarrow} v$ ou apenas por $u \rightarrow v$, quando não houver possibilidade de confusão. Observe, ainda, que permitimos 2-ciclos em nossa definição, isto é, que existam vértices $u, v$ tais que $u \rightarrow v$ e $v \rightarrow u$. Se um digrafo $G$ é tal que $u \rightarrow v$ implica $v \rightarrow u$, dizemos $G$ é um grafo ${ }^{1}$.

Definição 2.2 (torneio). Um torneio é um digrafo $(V, A)$ tal que para todo par $u, v \in V, u \neq v$, $\overrightarrow{u v} \in A \Leftrightarrow \overrightarrow{v u} \notin A$.

Em outras palavras, um torneio é um digrafo completo em que não há 2-ciclos.

Notação 2.3 (tamanho de um torneio). O tamanho $|T|$ de um torneio $T=(V, A)$ é dado pela cardinalidade de $V$. Ao denotarmos um torneio por $T_{n}$, estará implícito que seu tamanho é $n$.

Definição $2.4\left(\boldsymbol{\Gamma}^{+}, \boldsymbol{\Gamma}^{-}\right)$. Fixe um torneio $T=(V, A)$ e um vértice $u \in V$. Denotamos o conjunto de vértices dominados por $u$ por

$$
\Gamma^{+}(u)=\{v \in V: \overrightarrow{u v} \in A\}
$$

Analogamente, denotamos por $\Gamma^{-}(u)$ o conjunto de vértices que dominam $u$. Fixado um conjunto $W \subset V$, também definimos $\Gamma_{W}^{+}(u)=\Gamma^{+}(u) \cap W$ e $\Gamma_{W}^{-}(u)=\Gamma^{-}(u) \cap W$.

\footnotetext{
${ }^{1}$ Grafos não serão o foco deste trabalho, mas serão mencionados em certos momentos, uma vez que são um dos principais alvos de estudo em teste de propriedades. Recomendamos ao leitor não familiarizado com conceitos básicos de grafos consultar, por exemplo, [15].
} 
Definição 2.5 (grau, $\vec{e}$ ). O grau de saída e o grau de entrada de $u$ são dados, respectivamente, por $g^{+}(u)=\left|\Gamma^{+}(u)\right|$ e $g^{-}(u)=\left|\Gamma^{-}(u)\right|$. Para conjuntos $U, W \subset V$, também definimos $g_{W}^{+}(u)=\left|\Gamma_{W}^{+}(u)\right|$, $g_{W}^{-}(u)=\left|\Gamma_{W}^{-}(u)\right|$ e $\vec{e}(U, W)=\sum_{u \in U} g_{W}^{+}(u)$.

Definição 2.6 (isomorfismo). Dois torneios $T=(V, A)$ e $T^{\prime}=\left(V^{\prime}, A^{\prime}\right)$ são isomorfos se existe uma função bijetora $\phi: V \rightarrow V^{\prime}$ tal que $u \stackrel{T}{\rightarrow} v \Leftrightarrow \phi(u) \stackrel{T^{\prime}}{\rightarrow} \phi(v)$, isto é, se existe uma bijeção entre seus conjuntos de vértices que preserva todas as relações de dominância.

Definição 2.7 (torneio induzido). Seja $T=(V, A)$ um torneio e $W \subseteq V$ um subconjunto de vértices de $T$. O torneio induzido por $W$, denotado por $T[W]$, é o torneio $\left(W, A^{\prime}\right)$, onde

$$
A^{\prime}=\{\overrightarrow{x y} \in A: x, y \in W\}
$$

Definição 2.8 (caminho, ciclo, triângulo). Uma sequência de arcos da forma

$$
\overrightarrow{u_{1} u_{2}}, \overrightarrow{u_{2} u_{3}}, \ldots \overrightarrow{u_{k-1} u_{k}} \in A(T)
$$

determina um caminho de comprimento $k-1$ em um torneio $T$. Se, além disso, o arco $\overrightarrow{u_{k} u_{1}} \in A(T)$, então este arco unido aos arcos acima determinam um ciclo de comprimento $k$ em $T$. Um ciclo de tamanho três é chamado de triângulo dirigido.

Definição 2.9. Um torneio $T$ é dito transitivo ou acíclico se $T$ não contém nenhum ciclo.

O resultado abaixo mostra que torneios transitivos possuem uma estrutura muito simples.

Proposição 2.10 (cf. [27, Teorema 9]). Dado um torneio $T=(V, A)$, as seguintes afirmações são equivalentes:

(i) $T$ é transitivo.

(ii) T não contém triângulos dirigidos.

(iii) $\forall u, v, w \in V$, se $u \rightarrow v$ e $v \rightarrow w$, então $u \rightarrow w$.

(iv) existe uma enumeração $v_{1}, \ldots, v_{n}$ dos vértices de $T$ tal que $v_{i}$ domina $v_{j}$ para todo $1 \leq i<j \leq n . \diamond$

Demonstração. Vamos mostrar as seguintes implicações.

$(i) \Longrightarrow(i i)$. Trivial.

(ii) $\Longrightarrow$ (iii). Se $w \rightarrow u$, então $\overrightarrow{u v}, \overrightarrow{v w}, \overrightarrow{w u}$ formariam um triângulo dirigido.

(iii) $\Longrightarrow($ iv). Seja $n=|V|$. Se $n<3$ a afirmação é vacuamente verdadeira. Assuma $n \geq 3$ e fixe $v \in V \operatorname{com} g^{-}(v)>0$ e $g^{+}(v)>0$. Sejam $U=\Gamma^{-}(v), W=\Gamma^{+}(v), T_{-}=T[U]$ e $T_{+}=T[W]$. Por indução, existem enumerações $u_{1}, \ldots, u_{k} \in U$ e $w_{1}, \ldots, w_{n-k-1} \in W$ de vértices de $T_{-}$e $T_{+}$satisfazendo (iv). Mas, por hipótese, também não pode existir $u \in U$ e $w \in W$ tais que $w \rightarrow u$. Então a enumeração $u_{1}, \ldots, u_{k}, v, w_{1} \ldots, w_{n-k-1} \operatorname{satisfaz~}(i v)$.

$(i v) \Longrightarrow(i)$. T não pode conter um ciclo $\overrightarrow{v_{a} v_{b}}, \overrightarrow{v_{b} v_{c}}, \ldots, \overrightarrow{v_{p} v_{q}}, \overrightarrow{v_{q} v_{a}}$, caso contrário teríamos $a<b<$ $\cdots<q<a$, um absurdo. 
O último item da proposição anterior nos garante que todos os torneios transitivos de mesmo tamanho são isomorfos.

Notação 2.11. O torneio transitivo com $n$ vértices será denotado por $\pi_{n}$. Também denotamos por $\pi\left(v_{1}, \ldots, v_{n}\right)$ o torneio transitivo de tamanho $n$ com vértices $v_{1}, \ldots, v_{n}$ satisfazendo o item $(i v)$ da Proposição 2.10 .

Torneio transitivos desempenham um papel semelhante ao de grafos completos/vazios em Teoria dos Grafos. O resultado a seguir afirma que é possível encontrar cópias de torneios transitivos de tamanho arbitrário em todo torneio suficientemente grande.

Teorema 2.12 ([27, Teorema 10] Teorema de Ramsey para torneios). Para todo inteiro positivo $k$ existe $n=N_{2.12}(k)$ tal que para todo torneio $T=(V, A)$ com $|V| \geq n$, existe $W \subseteq V$ tal que $T[W]$ é um torneio transitivo de tamanho $k$.

Demonstração. Tome $n=2^{k}-1$. Para $k=1$ a afirmação é verdadeira. Assuma $k>1$ e que afirmação vale para conjuntos $W$ de tamanho $k-1$. Em um torneio $T_{n}$, deve existir ao menos um vértice $v$ com grau de saída pelo menos $(n-1) / 2=2^{k-1}-1$. Por hipótese de indução, $T\left[\Gamma^{+}(v)\right]$ contém $(k-1)$ vértices que induzem um torneio transitivo. Unidos a $v$, tais vértices induzem um torneio transitivo de tamanho $k$.

Definimos também um homomorfismo entre um torneio e um digrafo como uma função entre seus conjuntos de vértices que preserva relações de dominância. Tal função não precisa ser necessariamente injetora, contanto que vértices com a mesma imagem induzam um torneio transitivo.

Definição 2.13 (homomorfismo). Um homomorfismo entre um torneio $T$ e um digrafo $D$ é um função $\phi: V(T) \rightarrow V(D)$ satisfazendo as seguintes propriedades:

- se $u \stackrel{T}{\rightarrow} v$, então $\phi(u) \stackrel{D}{\rightarrow} \phi(v)$ ou $\phi(u)=\phi(v)$.

- para todo $x \in V(D), T\left[\phi^{-1}(x)\right]$ é um torneio transitivo.

Escrevemos $T \stackrel{\text { hom }}{\longrightarrow} D$ para indicar que uma tal função $\phi: V(T) \rightarrow V(D)$ existe.

Definição 2.14 (subgrafo, número de cópias). Seja $T$ um torneio e $D$ um digrafo. Dizemos que $T$ é subgrafo de $D$ se existe um homomorfismo injetor $\phi: V(T) \longmapsto V(D)$. Também usaremos o termo subtorneio quando $D$ for um torneio. O número de cópias de $T$ em $D$ é dado pelo número de homomorfismo injetores de $T$ em $D$. Também usamos o termo número de cópias não rotuladas quando contamos apenas os homomorfismos injetores cujas imagens são distintas (note que a razão entre essas duas últimas quantidades é no máximo $|T|$ !).

\section{$2.2 \quad$ Teste de Propriedade}

Definição 2.15 (Propriedade de torneios). Uma propriedade de torneios é um conjunto de torneios de mesmo tamanho, fechado por isomorfismo. Dizemos que um torneio $T$ satisfaz uma propriedade $\mathcal{P}$ se $T \in \mathcal{P}$. 
Estamos interessados em algoritmos que inspecionam apenas uma fração constante de um torneio $T_{n}$ de entrada e distinguem, com alta probabilidade, se $T$ satisfaz uma certa propriedade ou se está longe de satisfazê-la. Antes de mais nada, é preciso fixar um modelo, isto é, definir uma noção de distância de torneios a propriedades assim como especificar de que forma tais algoritmos podem acessar a entrada Utilizamos um modelo natural, análogo ao dito modelo denso para grafos [21], também conhecido como modelo da matriz de adjacência.

Definição 2.16 ( $\epsilon$-longe, $\epsilon$-perto, distância). Dizemos que um torneio $T_{n}$ é/está $\epsilon$-longe de uma propriedade $\mathcal{P}$ se é necessário inverter a orientação de pelo menos $\epsilon n^{2}$ arcos de $T_{n}$ para fazê-lo satisfazer $\mathcal{P}$. Caso contrário, dizemos que $T_{n}$ está $\epsilon$-perto de $\mathcal{P}$. Definimos a distância de $T_{n}$ a $\mathcal{P}$ como o menor $\epsilon$ tal que $T_{n}$ está $\epsilon$-longe de $\mathcal{P}$.

Ao trabalharmos com algoritmos sublineares, também é necessário especificar como os objetos de entrada são representados, uma vez que tais algoritmos não podem sequer ler toda a entrada. Para tanto, consideraremos algoritmos que acessam o torneio de entrada por meio de um oráculo.

Definição 2.17 (oráculo). Um oráculo associado a um torneio $T=(V, A)$ é um objeto que recebe consultas formadas por pares $u \neq v$ de vértices em $V$ e devolve o valor de $f(u, v)$, onde a $f: V \times V \backslash\{(v, v): v \in V\} \rightarrow A$ é a função dada por

$$
f(u, v)=\left\{\begin{array}{l}
\overrightarrow{u v}, \text { se } u \rightarrow v . \\
\overrightarrow{v u}, \text { se } v \rightarrow u
\end{array}\right.
$$

Notamos que no estudo de teste de propriedades em grafos, existem outros modelos que podem ser mais propícios em casos específicos (o modelo denso, por exemplo, não é apropriado para o estudo de grafos que possuem uma quantidade subquadrática de arestas). O modelo da lista de incidência [19] representa grafos que têm grau máximo limitado por uma constante $d$, permitindo consultas a uma função $g: V \times[d] \rightarrow V \cup\{\perp\}$ que indica o $i$-ésimo vizinho de um dado vértice. Nesse modelo, dois grafos estão $\epsilon$-longe se a cardinalidade da diferença simétrica entre seus conjuntos de arestas supera $\epsilon d n / 2$. O modelo geral [28] tenta dissociar a forma com que as consultas são feitas (assim como a definição de distância) da representação interna dos grafos. Nesse modelo, a distância entre dois grafos $(V, E),\left(V^{\prime}, E^{\prime}\right)$ é dada por $\frac{\left|E \Delta E^{\prime}\right|}{\max \left\{|E|,\left|E^{\prime}\right|\right\}}$; ou seja, a distância é normalizada pelo próprio número de arestas dos grafos em questão e não por um limitante superior, como $n^{2}$ ou $d N$. Esse modelo suporta, ainda, ambos os tipos de consultas dos modelos anteriores. Esses modelos não possuem, contudo, versões que pareçam apropriadas para torneios.

Definição 2.18 ( $\epsilon$-testador, $\boldsymbol{q}(\mathcal{A})$ ). Um $\epsilon$-testador $\mathcal{A}$ para uma propriedade de torneios $\mathcal{P}$ é um algoritmo de decisão probabilístico que recebe um inteiro $n$ e um oráculo associado a um torneio $T_{n}$ e que satisfaz as seguintes condições:

1. Se $T_{n} \in \mathcal{P}$, então $\mathcal{A}$ aceita $T_{n}$ com probabilidade pelo menos $\frac{2}{3}$.

2. Se $T_{n}$ está $\epsilon$-longe de $\mathcal{P}$, então $\mathcal{A}$ rejeita $T_{n}$ com probabilidade pelo menos $\frac{2}{3}$.

3. O número máximo $q(\mathcal{A})$ de consultas que $\mathcal{A}$ faz ao oráculo independe de $n$.

Ademais, se a primeira condição é satisfeita com probabilidade 1, dizemos que $\mathcal{A}$ tem erro-unilateral. $\diamond$ 
Em outras palavras, um $\epsilon$-testador para $\mathcal{P}$ é um algoritmo probabilístico que distingue, com probabilidade pelo menos $\frac{2}{3}$, torneios que satisfazem $\mathcal{P}$ de torneios que estão $\epsilon$-longe de $\mathcal{P}$.

Note que a Definição 2.18 não impõe nenhuma condição para o caso de torneios a uma distância estritamente entre 0 e $\epsilon$ a $\mathcal{P}$. Como consequência, "conter um triângulo dirigido" ou "conter um ciclo que passa por todos os vértices" são exemplos de propriedades que admitem um $\epsilon$-testador trivial (para qualquer $\epsilon$ ), que simplesmente aceita qualquer entrada (suficientemente grande). De fato, a primeira condição da Definição 2.18 valerá com probabilidade 1, e a segunda será vacuamente verdadeira, uma vez que não há torneios $\epsilon$-longe de tais propriedades se $n$ for grande o suficiente.

Observação 2.19. Ao estudar $\epsilon$-testadores, sempre podemos assumir que os torneios de entrada são suficientemente grandes uma vez que, para entradas de tamanho limitado, um testador pode simplesmente verificar por força bruta se o torneio satisfaz $\mathcal{P}$, após consultar todos os seus arcos. $\diamond$

Definição 2.20 (propriedade testável). Uma propriedade $\mathcal{P}$ é dita testável [com erro-unilateral] se para qualquer $0<\epsilon<1$ existe um $\epsilon$-testador [com erro-unilateral ] para $\mathcal{P}$.

Note como a terceira condição da Definição 2.18 implica que todo $\epsilon$-testador consome tempo constante em relação ao tamanho da entrada. É comum encontrar o termo teste de propriedade sendo usado em um contexto mais amplo, em que se consideram algoritmos que consomem tempo não necessariamente constante, mas sublinear no tamanho da entrada. Nesse caso, é empregado o termo fortemente testável para se referir a propriedades como na definição acima. Contudo, boa parte do interesse da área se concentra no estudo de propriedades testáveis em tempo constante, de modo que esse será o foco deste trabalho.

O consumo de tempo de um testador, embora constante em função do tamanho do torneio de entrada, pode ser classificado de acordo com a dependência com o parâmetro de erro $\epsilon$.

Definição 2.21 (propriedade facilmente testável). Uma propriedade $\mathcal{P}$ é facilmente testável se para qualquer $0<\epsilon<1$, existem um $\epsilon$-testador com erro-unilateral $\mathcal{A}_{\epsilon}$ para $\mathcal{P}$ e $q\left(\mathcal{A}_{\epsilon}\right)=$ $\operatorname{poli}\left(\epsilon^{-1}\right)$.

Em um primeiro momento, poderia parecer mais natural considerar o parâmetro $\epsilon$ como parte da entrada, na definição de um testador. Essa diferença aparentemente sutil teria um grande impacto na caracterização de propriedades testáveis unilateralmente: veremos que existem propriedades que, embora admitam $\epsilon$-testadores (unilaterais) para todo $\epsilon$, exigem um número de consultas ao oráculo que não é sequer computável como função de $\epsilon$. Em outras palavras é possível que uma propriedade admita $\epsilon$-testadores para todo $\epsilon$ fixo, mas que não seja possível combiná-los em um único algoritmo que recebe $\epsilon$ como entrada.

Definição 2.22 (Testador não-adaptativo). Um $\epsilon$-testador $\mathcal{A}$ para uma propriedade $\mathcal{P}$ é dito não-adaptativo se existe uma função $q:[0,1] \times \mathbb{N} \rightarrow \mathbb{N}$ tal que $\mathcal{A}$ procede da seguinte maneira ao processar uma entrada $T_{n}=(V, A)$.

- Primeiro, $\mathcal{A}$ escolhe, uniformemente ao acaso, um conjunto $Q \subseteq V$ de tamanho $q:=q(\epsilon, n)$.

- Em seguida, $\mathcal{A}$ faz $q(q-1) / 2$ consultas ao oráculo para obter o subtorneio $T_{n}[Q]$ induzido por $Q$. 
- Por fim, $\mathcal{A}$ aceita ou rejeita a entrada tendo acesso somente ao subtorneio $T_{n}[Q]$ e ao valor de $n$, isto é, sem fazer mais nenhuma consulta adicional ao oráculo.

Note, na definição acima, que o tamanho $q$ da consulta é também função do tamanho $n$ do torneio de entrada (apenas o número máximo de consultas deve independer de $n$ ) e, principalmente, que a decisão final do testador também pode depender de $n$. Essa dependência é permitida para que possam existir testadores não-adaptativos para propriedades que dependem explicitamente do valor de $n$, tais como: "ser transitivo se $n$ é primo ou livre de ciclos de tamanho 5 caso contrário" ou mesmo "ter tamanho ímpar". Na prática, propriedades "artificiais" como essas serão desconsideradas (ver Observação 2.24).

O resultado a seguir nos permite assumir que todos os testadores são não-adaptativos.

Teorema $2.23\left([22,23]_{\text {gr }}\right)$. Toda propriedade testável $\mathcal{P}$ possui um $\epsilon$-testador não-adaptativo $\mathcal{B}$, para todo $\epsilon>0$. Ademais,

- se $\mathcal{P}$ é testável com erro-unilateral, então podemos supor que $\mathcal{B}$ tem erro-unilateral.

- se $q(\mathcal{P})=\operatorname{poli}\left(\epsilon^{-1}\right)$, ent $\tilde{a} o q(\mathcal{B})=\operatorname{poli}\left(\epsilon^{-1}\right)$.

Demonstração. Seja $\mathcal{P}$ uma propriedade testável e $\mathcal{A}$ um testador qualquer para $\mathcal{P}$. Primeiro, transformamos $\mathcal{A} \mathrm{em}$ um testador revelador de vértices. Um testador é revelador de vértices se podemos dividi-lo em iterações satisfazendo o seguinte: a cada iteração, um novo vértice $v$ é selecionado e são feitas (apenas) consultas a todos os pares da forma $(u, v)$, onde $u$ é um vértice selecionado em uma iteração anterior. É evidente que podemos emular $\mathcal{A}$ por meio de um testador revelador de vértices $\mathcal{A}_{R}$ que, a cada consulta $(u, v)$ de $\mathcal{A}$, revela os vértices $u$ e $v$ (caso ainda não tenham sido revelados).

Agora, considere o algoritmo $\mathcal{A}_{U}$ descrito a seguir. Primeiramente, $\mathcal{A}_{U}$ escolhe, uniformemente ao acaso, uma permutação $\sigma$ do conjunto de vértices do torneio de entrada. Em seguida, $\mathcal{A}_{U}$ emula $\mathcal{A}_{R}$, da seguinte maneira: toda consulta $(u, v)$ requisitada ao oráculo por $\mathcal{A}_{R}$ é alimentada com a resposta da consulta $(\sigma(u), \sigma(v))$.

Note que aplicar o algoritmo $\mathcal{A}_{U}$ a um torneio $T$ é o mesmo que aplicar $\mathcal{A}_{R}$ ao torneio $\sigma(T)$. Portanto, $\mathcal{A}_{U}$ ainda é um testador (revelador de vértices) para $\mathcal{P}$, uma vez que propriedades são fechadas por isomorfismo.

Fixe $i \geq 1$ e suponha que $\mathcal{A}_{U}$ já tenha revelado os vértices $u_{1}, \ldots, u_{i-1}$ nas primeiras $i-1$ iterações e que esteja prestes a revelar um novo vértice $u_{i}$. Note que o vértice $r_{i}$ a ser revelado na $i$-ésima iteração de $\mathcal{A}_{R}$ depende apenas ${ }^{2}$ dos vértices $r_{1}, \ldots, r_{i-1}$ revelados nas iterações anteriores. Então devemos ter

$$
\mathbb{P}\left(u_{i}=x \mid u_{1}, \ldots, u_{i-1}\right)=\mathbb{P}\left(\sigma\left(r_{i}\right)=x \mid \sigma\left(r_{1}\right)=u_{1}, \ldots, \sigma\left(r_{i-1}\right)=u_{i-1}\right)=\frac{1}{n-i+1},
$$

ou seja, os vértices revelados por $\mathcal{A}_{U}$ têm distribuição uniforme sobre os vértices ainda não revelados. Então podemos modificar $\mathcal{A}_{U}$ de forma que os vértices sejam escolhidos uniformemente ao acaso no início do algoritmo, obtendo assim um algoritmo não-adaptativo.

Terminamos a demonstração observando que $q\left(\mathcal{A}_{u}\right)=q\left(\mathcal{A}_{R}\right) \leq 2(q(\mathcal{A}))^{2}$.

\footnotetext{
${ }^{2}$ é verdade que $r_{i}$ também pode depender de possíveis decisões aleatórias feitas por $\mathcal{A}_{R}$; nesse caso, basta reiniciar o argumento fixando uma fita de bits aleatórios usados por $\mathcal{A}_{R}$ para realizar tais decisões.
} 
Observação 2.24 (redefinição de propriedade testável). O resultado acima nos permite redefinir propriedades testáveis como aquelas que possuem, para cada $\epsilon$, um $\epsilon$-testador não-adaptativo. A partir daqui, contudo, exigiremos que tais $\epsilon$-testadores não dependam de $n$ para calcular o número de consultas ou para fazer a decisão final a partir do subtorneio amostrado. As propriedades testáveis estudadas na literatura admitem, todas, testadores dessa forma. Em outras palavras, o único impacto dessa restrição adicional é o de excluir aquelas propriedades "artificiais" mencionadas anteriormente.

Observação 2.25. Ao analisar testadores não-adaptativos, podemos supor que cada um dos $q$ vértices da amostra é escolhido uniforme e independentemente ao acaso. De fato, a probabilidade de um conjunto $Q$ fixo de cardinalidade $q$ ser escolhido nesse processo (dada por $q ! n^{-q}$ ) é arbitrariamente próxima à probabilidade da distribuição real $\left(\left(\begin{array}{l}n \\ q\end{array}\right)^{-1}\right)$ para $n$ suficientemente grande.

\subsection{Exemplo: transitividade é uma propriedade testável}

O objetivo desta seção é mostrar que a propriedade "ser transitivo" é um exemplo de uma propriedade testável de torneios. Mais especificamente, queremos mostrar o seguinte.

Teorema 2.26 ([11]). Seja Trans $:=\left\{T_{n}: T_{n}\right.$ é transitivo $\}$. Então Trans é uma propriedade de torneios facilmente testável.

Devemos mostrar que, dados um torneio $T$ e um parâmetro de erro $\epsilon$, existe um algoritmo como na Definição 2.18. O algoritmo em questão será um algoritmo não-adaptativo que amostra (de maneira uniforme e independente) um subconjunto $Q$ de vértices de $T$, de tamanho $q=\operatorname{poli}\left(\epsilon^{-1}\right)$, e considera o subtorneio induzido $T[Q]$, onde $Q$ é o conjunto dos vértices amostrados. A seguir, o algoritmo simplesmente aceita a entrada se $T[Q]$ for transitivo ou rejeita-a caso contrário. Se $T$ for transitivo então o subtorneio amostrado não conterá ciclos, de modo que o algoritmo de fato aceitará $T$ com probabilidade 1. Assim, só é preciso mostrar que se $T$ é $\epsilon$-longe de ser transitivo, então $Q$ contém um ciclo com probabilidade pelo menos $\frac{2}{3}$.

Seja $T_{n}=(V, A)$ um torneio. Primeiro, mostramos que se $T_{n}$ está longe de ser transitivo, então $T_{n}$ contém um conjunto $W$ grande, em que cada vértice de $W$ tem grau de saída alto em $W$.

Lema 2.27. Se $T_{n}$ está $\epsilon$-longe de Trans, então existe $W \subseteq V,|W| \geq \sqrt{\frac{\epsilon}{2}} n$ tal que para todo $w \in W, g_{W}^{+}(w)>\frac{1}{2} \epsilon n$.

Demonstração. Suponha que para um dado torneio $T_{n}$, não exista um conjunto $W$ como no enunciado. Queremos provar que $T_{n}$ está $\epsilon$-perto de Trans. Considere o seguinte procedimento:

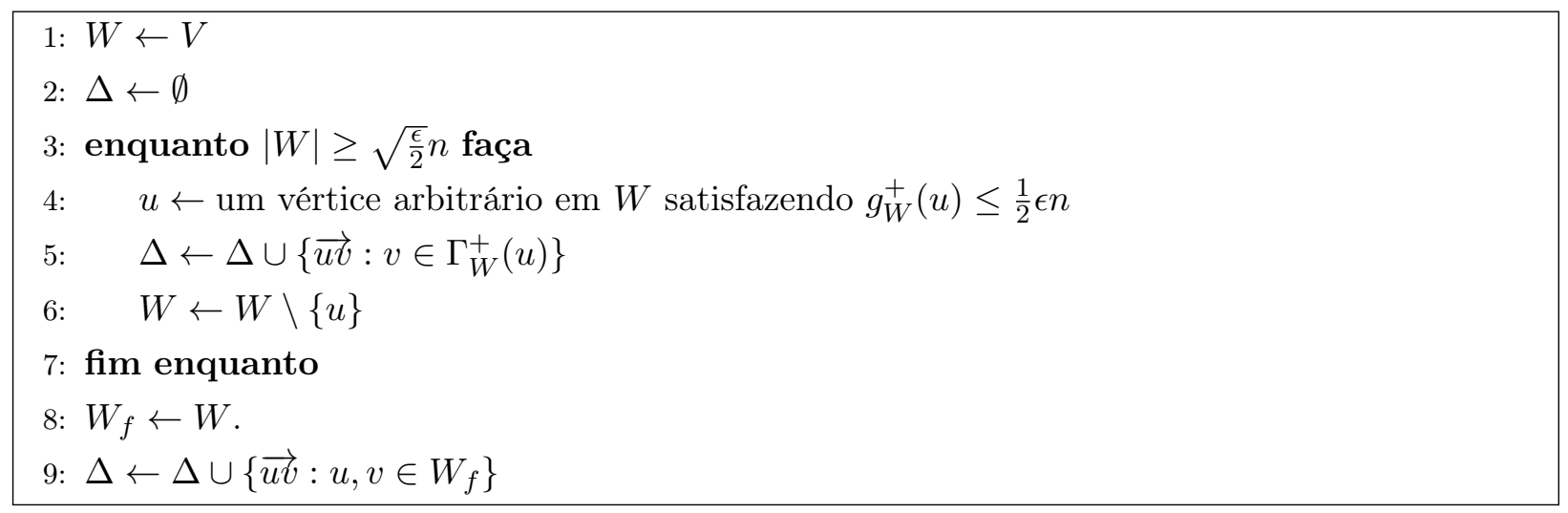


Seja $U=\left\{u_{i}\right\}_{1 \leq i \leq k}$, onde $u_{i}$ é o vértice $u$ escolhido na $i$-ésima iteração da linha 4 e $k=$ $\max \left\{0, n-\left\lfloor\sqrt{\frac{\epsilon}{2}} n\right\rfloor\right\}$. Defina, ainda, $w_{1}, \ldots, w_{n-k}$ como sendo os vértices de $W_{f}$, indexados em uma ordem arbitrária e considere o torneio transitivo $\pi=\pi\left(w_{1}, \ldots, w_{n-k}, u_{k}, u_{k-1}, \ldots, u_{1}\right)$.

Afirmamos que $\Delta\left(T_{n}, \pi\right) \subseteq \Delta$, onde $\Delta\left(T_{n}, \pi\right)$ é o conjunto de $\operatorname{arcos} \overrightarrow{x y} \in A\left(T_{n}\right)$ tais que $\overrightarrow{y x} \in \pi$. De fato, para todo arco $\overrightarrow{u_{i} u_{j}} \notin \Delta$, deve valer que $i>j$ - caso contrário $\overrightarrow{u_{i} u_{j}}$ teria sido adicionado a $\Delta$ na iteração $i$ - o que implica que o sentido deste arco está de acordo com $\pi$, ou seja, que $\overrightarrow{u_{i} u_{j}} \notin \Delta\left(T_{n}, \pi\right)$.

Por outro lado, acrescentamos a $\Delta$ no máximo $\frac{1}{2} \epsilon n$ arcos a cada iteração da linha 5 e menos que $\left|W_{f}\right|^{2}=\frac{1}{2} \epsilon n^{2}$ arcos na linha 9. Portanto, concluímos que $|\Delta|<\epsilon n^{2}$, isto é, que $T_{n}$ não está $\epsilon$-longe de Trans.

A seguir, mostramos que ao amostrarmos vértices de um tal conjunto $W$, o subtorneio induzido contém ciclos com alta probabilidade.

Lema 2.28. Sejam $0<\gamma<1$ e $W \subseteq V$ um subconjunto de vértices de $T_{n}$ tal que $g_{W}^{+}(w) \geq \gamma|W|$ para todo $w \in W$. Seja $Q \subseteq W$ um conjunto de tamanho $m=m_{2.28}(\gamma)=\operatorname{poli}\left(\gamma^{-1}\right)$ de vértices de $W$, escolhido uniformemente ao acaso. Então o subtorneio induzido $T_{n}[Q]$ contém um ciclo com probabilidade pelo menos $\frac{7}{8}$.

Demonstração. Tome $m=\frac{10 \ln (1 / \gamma)}{\gamma}+1$. Vamos mostrar que com probabilidade pelo menos $\frac{7}{8}$, $g_{Q}^{+}(u)>0$ para todo $u \in Q$, o que implicará que $T_{n}[Q]$ contém algum ciclo (uma vez que não há sorvedouros em $\left.T_{n}[Q]\right)$.

Fixado um vértice $u \in Q$, temos que

$$
\mathbb{P}\left(g_{Q}^{+}(u)=0\right) \leq(1-\gamma)^{m-1}<e^{-(m-1) \gamma}=\gamma^{10}
$$

Pela cota da união, concluímos que

$$
\mathbb{P}\left(\forall u \in Q, g_{Q}^{+}(u)=0\right)<\sum_{i=1}^{m} \gamma^{10}=\left(\frac{10 \ln (1 / \gamma)}{\gamma}+1\right) \gamma^{10}
$$

Como $\gamma \leq \frac{1}{2}$, a probabilidade acima é menor que $\frac{1}{8}$, como desejado.

Demonstração do Teorema 2.26. Considere um testador que amostra um conjunto de vértices $Q$ de tamanho $q=4 m \epsilon^{-1}$, onde $m=m_{2.28}\left(\frac{1}{2} \epsilon\right)$, e procede como descrito no início da seção. Se $T_{n}$ é transitivo, o algoritmo claramente o aceita com probabilidade 1 . Suponha então que $T_{n}$ é $\epsilon$-longe de ser transitivo. Então pelo Lema 2.27 existe um conjunto $W \subseteq V,|W| \geq \sqrt{\frac{\epsilon}{2}} n$, tal que para todo $w \in W, g_{W}^{+}(w)>\frac{\epsilon}{2} n$.

Seja $n_{W}$ o número de vértices amostrados que pertencem a $W$. Como $q \geq \frac{2 m|V|}{|W|}$, o valor de $n_{W}$ é, em média, pelo menos $2 m$. Mais do que isso, podemos usar uma cota de Chernoff (Teorema A.2) para obter que $\mathbb{P}\left(n_{W}<m\right)=\mathbb{P}\left(n_{W}<\left(1-\frac{1}{2}\right) 2 m\right) \leq e^{-\frac{1}{4} m}$. Portanto, para $m \geq 9$, a probabilidade de pelo menos $m$ vértices de $Q$ pertencerem a $W$ é maior que $\frac{7}{8}$.

Assumindo que o evento $\left[n_{W} \geq m\right]$ de fato ocorre, podemos tomar $\gamma=\frac{\epsilon}{2}$ e aplicar o Lema 2.28 para concluir que $T_{n}[Q]$ contém um ciclo, com probabilidade $\frac{7}{8}$.

Assim, o algoritmo proposto de fato encontra um ciclo em $T_{n}[Q]$ com probabilidade pelo menos $1-\frac{1}{4}>\frac{2}{3}$ 


\section{Capítulo 3}

\section{Inevitabilidade e Abundância de subtorneios}

Definição 3.1 (Livre $\left(\boldsymbol{T}_{\boldsymbol{h}}\right)$ ). Dizemos que um torneio $T$ é livre de um torneio $T_{h}$ se $T$ não contém nenhuma cópia de $T_{h}$. Definimos a propriedade Livre $\left(T_{h}\right)$ como o conjunto de torneios que são livres de $T_{h}$.

Mostraremos no capítulo 4 que a propriedade $\operatorname{Livre}\left(T_{h}\right)$ é testável para qualquer torneio $T_{h}$. Neste capítulo, introduzimos boa parte das ferramentas necessárias para provar tal resultado, além de usá-las para apresentar uma versão mais fraca do mesmo, enunciada a seguir.

Definição 3.2 (transversal). Fixado um torneio $T_{h}$, uma transversal de $T_{h}$ em um torneio $T=$ $(V, A)$ é um subconjunto $S \subseteq A$ de $\operatorname{arcos}$ de $T$ tal que toda cópia de $T_{h}$ em $T$ contém arcos em $S . \diamond$

Definição 3.3 ( $\epsilon$-inevitável). Dizemos que um torneio $T_{h}$ é $\epsilon$-inevitável em um torneio $T_{n}$ se toda transversal de $T_{h}$ em $T_{n}$ tem tamanho pelo menos $\epsilon n^{2}$.

Definição 3.4 ( $\delta$-abundante). Dizemos que um torneio $T_{h}$ é $\delta$-abundante em um torneio $T_{n}$ se $T_{n}$ contém pelo menos $\delta n^{h}$ cópias de $T_{h}$.

Teorema 3.5 ([2] $\left.]_{\text {gr }}\right)$. Para todo inteiro $h$ e $0<\epsilon<1$ existe $\delta=\delta_{3.5}(\epsilon, h)$ tal que se $T_{h}$ é $\epsilon$ inevitável em um torneio $T$, então $T_{h}$ é $\delta$-abundante em $T$.

O resultado acima dá origem a um algoritmo capaz de distinguir, em tempo constante e com alta probabilidade, entre torneios $T$ livres de $T_{h}$ e torneios $T$ em que $T_{h}$ é $\epsilon$-inevitável. De fato, podemos simplesmente verificar se um conjunto de vértices amostrado de $T$ (de um certo tamanho constante) induz uma cópia de $T_{h}$, uma vez que o resultado acima nos garante que encontraremos alguma com alta probabilidade se $T_{h}$ for inevitável em $T$ (e nenhuma será encontrada em torneios livres de $T_{h}$ ).

Ao trabalharmos com grafos, um resultado análogo ao Teorema 3.5 já é suficiente para mostrar que a propriedade $\mathcal{P}=$ "ser livre de cópias ${ }^{1}$ de um subgrafo $H$ " (análoga a Livre $\left(T_{h}\right)$ ) é testável. Isso é verdade pois a noção de distância no caso de grafos envolve adição ou remoção de arestas

\footnotetext{
${ }^{1}$ não necessariamente induzidas.
} 
e, portanto, estar $\epsilon$-longe de tal propriedade implica $H$ ser $\epsilon$-inevitável. Infelizmente, essa relação entre inevitabilidade e distância não é válida para torneios: é possível que um torneio $\epsilon$-longe de Livre $\left(T_{h}\right)$ tenha uma $\epsilon$-transversal de $T_{h}$ de tamanho menor que $\epsilon n^{2}$, uma vez que a inversão de arcos de tal transversal pode dar origem a novas cópias de $T_{h}$.

\subsection{Regularidade em torneios}

Um resultado extensivamente usado em diversas áreas [25], é o chamado Lema de Regularidade de Szemerédi [30] que, essencialmente, afirma que todo grafo suficientemente grande admite uma partição regular, isto é, uma divisão de seus vértices em um número limitado de partes, de forma que quase todo o par de partes se comporta de maneira semelhante a um grafo bipartido aleatório.

No contexto de teste de propriedades em grafos, Alon e co-autores mostraram em [4] que tal lema não é apenas uma ferramenta, mas está intrinsecamente relacionado ao conceito de testabilidade. Tais autores mostraram, essencialmente ${ }^{2}$, que as propriedades testáveis de grafos são exatamente aquelas formadas pelos grafos que admitem uma partição regular pertencente a um dado conjunto finito de partições regulares.

Existem generalizações do Lema de Regularidade para diversos objetos, como grafos com arestas coloridas, grafos dirigidos e hipergrafos (ver, por exemplo, [25]). Apresentamos aqui uma versão específica para torneios, cuja demonstração é baseada na versão para grafos contida em [10][Capítulo $17]$.

\subsubsection{Definições iniciais}

Definição 3.6 $(\vec{e}(\boldsymbol{X}, \boldsymbol{Y}), \overrightarrow{\boldsymbol{d}}(\boldsymbol{X}, \boldsymbol{Y}))$. Dado um torneio $T=(V, A)$ e conjuntos $X, Y \subseteq V$ disjuntos, definimos e a densidade do par $(X, Y)$ como

$$
\vec{d}(X, Y)=\frac{\vec{e}(X, Y)}{|X||Y|}
$$

onde

$$
\vec{e}(X, Y)=|\{\overrightarrow{x y}: x \in X, y \in Y\}| .
$$

Definição 3.7 (par regular). Sejam $T=(V, A)$ um torneio, $\gamma \in \mathbb{R}$, e $X, Y \subset V$ dois conjuntos disjuntos de $V$. Dizemos que o par $(X, Y)$ é $\gamma$-regular se para quaisquer $X^{\prime} \subseteq X$ e $Y^{\prime} \subseteq Y$, satisfazendo $\left|X^{\prime}\right| \geq \gamma|X|$ e $\left|Y^{\prime}\right| \geq \gamma|Y|$ vale que $\vec{d}\left(X^{\prime}, Y^{\prime}\right)=\vec{d}(X, Y) \pm \gamma$. Dizemos que um par $(X, Y)$ é $\gamma$-irregular se a condição acima é falsa. Dizemos, ainda, que um par $(X, Y)$ é apenas regular ou irregular quando o parâmetro $\gamma$ envolvido estiver implícito ou não for importante no contexto.

Em outras palavras, um par $(X, Y)$ é $\gamma$-regular se a densidade $\vec{d}(X, Y)$ é herdada, a menos por um erro $\gamma$, por todos os pares de subconjuntos de cardinalidade substancial. Note que sem essa restrição de tamanho sobre os subconjuntos $X^{\prime}$ e $Y^{\prime}$, a definição seria vazia (se $X^{\prime}$ e $Y^{\prime}$ são conjuntos unitários, por exemplo, a densidade entre eles só pode ser 0 ou 1). Note também que a definição de par regular é simétrica, isto é, $(X, Y)$ é $\gamma$-regular se e só se $(Y, X)$ é $\gamma$-regular.

\footnotetext{
${ }^{2}$ consultar capítulo 7 para uma afirmação mais precisa.
} 
Se todos os arcos que cruzam a partição $(X, Y)$ tiverem a mesma orientação, então $(X, Y)$ é um exemplo trivial de par regular. Na verdade, é fácil ver que se $\vec{d}(X, Y)<\gamma^{3}$ ou $\vec{d}(X, Y)>\left(1-\gamma^{3}\right)$, então $(X, Y)$ é $\gamma$-regular. Por outro lado, a construção explícita de pares regulares não triviais não é uma tarefa imediata. O exemplo abaixo atesta que tais pares, de fato, existem e também ilustra a relação entre regularidade e torneios aleatórios.

Exemplo 3.8. Seja $T=(X \sqcup Y, A),|X|=|Y|=n$, um torneio em que para cada $x \in X$ e $y \in Y$, fazemos $x$ dominar $y$ com probabilidade $p$, de maneira independente das demais escolhas aleatórias. Então para qualquer $\gamma>0$ fixo, o par $(X, Y)$ é $\gamma$-regular assintoticamente quase certamente.

Demonstração. Fixe $X^{\prime} \subseteq X$ e $Y^{\prime} \subseteq Y$ satisfazendo $\left|X^{\prime}\right| \geq \gamma n$ e $Y^{\prime} \geq \gamma n$. Primeiro, usamos uma cota de Chernoff (Teorema A.2) para mostrar que

$$
\mathbb{P}(|\vec{d}(X, Y)-p|>\gamma / 2) \leq 2 e^{-\frac{\left(\gamma n^{2} / 2\right)^{2}}{3\left(n^{2} p\right)}}=2 e^{-\frac{\gamma^{2} n^{2}}{12 p}}
$$

Aplicando a mesma desigualdade para o par $\left(X^{\prime}, Y^{\prime}\right)$, temos

$$
\left.\mathbb{P}\left(\left|\vec{d}\left(X^{\prime}, Y^{\prime}\right)-p\right|\right)>\gamma / 2\right) \leq 2 e^{-\frac{\gamma^{4} n^{2}}{12 p}}
$$

e portanto segue da desigualdade triangular que

$$
\mathbb{P}\left(\left|\vec{d}(X, Y)-\vec{d}\left(X^{\prime}, Y^{\prime}\right)\right|>\epsilon\right) \leq 4 e^{-\frac{\gamma^{4} n^{2}}{12 p}}
$$

Pela cota da união, a probabilidade de $(X, Y)$ ser $\gamma$-irregular é no máximo

$$
2^{2 n} 4 e^{-\frac{\gamma^{4} n^{2}}{12 p}} \leq 4 e^{-\frac{\gamma^{4} n^{2}-2 n}{12 p}} \longrightarrow 0
$$

quando $n \longrightarrow \infty$, como desejado.

A seguir, mostramos duas propriedades úteis de pares regulares, que são usadas em vários contextos (ver, por exemplo, [25] para o caso de grafos). A primeira afirma que a regularidade é de certa forma "herdada" por subconjuntos de tamanho considerável. A segunda afirma que, dado um par $(X, Y)$ regular, quase todos os vértices de $X$ dominam muitos vértices de um conjunto consideravelmente grande de $Y$.

Proposição 3.9. Seja $(X, Y)$ um par $\gamma$-regular de densidade $\vec{d}(X, Y)=\eta$ e $X_{1} \subseteq X, Y_{1} \subseteq Y$ conjuntos satisfazendo $\left|X_{1}\right| \geq \epsilon|X|$ e $\left|Y_{1}\right| \geq \epsilon|Y|$, com $\epsilon \geq \gamma$. Então o par $\left(X_{1}, Y_{1}\right)$ é $\max \left\{2 \gamma, \frac{\gamma}{\epsilon}\right\}$ regular (com densidade $\eta \pm \gamma$ ).

Demonstração. Sejam $X_{2} \subseteq X_{1}$ e $Y_{2} \subseteq Y_{1}$ subconjuntos de tamanho pelo menos $(\gamma / \epsilon)\left|X_{1}\right|$ e $(\gamma / \epsilon)\left|Y_{1}\right|$, respectivamente. Então $\left|X_{2}\right| \geq \gamma|X|$ e $\left|Y_{2}\right| \geq \gamma|Y|$ e, como $(X, Y)$ é $\gamma$-regular, segue que:

$$
\vec{d}\left(X_{2}, Y_{2}\right)=\vec{d}(X, Y) \pm \gamma=\left(\vec{d}\left(X_{1}, Y_{1}\right) \pm \gamma\right) \pm \gamma=\vec{d}\left(X_{1}, Y_{1}\right) \pm 2 \gamma
$$

o que atesta a $\max \left\{2 \gamma, \frac{\gamma}{\epsilon}\right\}$-regularidade de $\left(X_{1}, Y_{1}\right)$.

Proposição 3.10. Seja $(X, Y)$ um par $\gamma$-regular com densidade $\vec{d}(X, Y)=\eta$. Considere um conjunto $Y^{\prime} \subseteq Y$ tal que $\left|Y^{\prime}\right| \geq \gamma|Y|$. Então no máximo $\gamma|X|$ dos vértices de $X$ dominam menos que $(\eta-\gamma)\left|Y^{\prime}\right|$ vértices em $Y^{\prime}$. 
Demonstração. Suponha por absurdo que exista um conjunto $X^{\prime} \subseteq X$ de tamanho maior que $\gamma|X|$ que contradiga a asserção acima. Então o par $\left(X^{\prime}, Y^{\prime}\right)$ tem menos que $(\eta-\gamma)\left|X^{\prime}\right|\left|Y^{\prime}\right|$ arestas e é portanto uma testemunha de que $(X, Y)$ não é $\gamma$-regular.

\subsubsection{Lema de regularidade}

Definição 3.11 (partição $\gamma$-regular). Uma partição $\gamma$-regular de um torneio $T_{n}=(V, A)$ é uma partição $\mathcal{V}=\left\{V_{0}, V_{1}, \ldots, V_{m}\right\}$ de $V$ satisfazendo as seguintes propriedades:

(i) Todos a menos de no máximo $\gamma m^{2}$ dos pares $\left(V_{i}, V_{j}\right), 1 \leq i<j \leq m$, são $\gamma$-regulares.

(ii) $\left|V_{1}\right|=\left|V_{2}\right|=\cdots=\left|V_{m}\right|$

(iii) $\left|V_{0}\right| \leq \gamma n$.

O conjunto $V_{0}$ é chamado de parte excepcional da partição regular.

Notação 3.12 (parte excepcional). Ao dizermos que um conjunto $V_{0}$ é uma parte excepcional de uma partição $\mathcal{V}$, estará implícito que $V_{0}$ deve ser considerado formalmente como uma coleção de $\left|V_{0}\right|$ partes unitárias (cuja união é $V_{0}$ ), todas contidas em $\mathcal{V}$. Considerar $V_{0}$ como uma parte de $\mathcal{V}$ é apenas um abuso que visa não sobrecarregar a notação.

O Lema de Regularidade afirma que podemos obter uma partição regular de qualquer torneio $T_{n}$ (suficientemente grande) com um número de partes que independe de $n$ e que pode ser limitado inferiormente (o que nos permite limitar o número de arcos internos a cada parte).

Teorema 3.13 ([7] $]_{g r}$ Lema de Regularidade com parte excepcional). Para todo $\gamma>0 e$ inteiro $\mu$, existe um inteiro $M=M_{3.13}(\gamma, \mu)$ tal que todo torneio $T$ de tamanho pelo menos $M$ admite uma partição $\gamma$-regular $\left\{V_{0}, V_{1}, \ldots, V_{m}\right\} \operatorname{com} \mu \leq m \leq M$.

Para demonstrar o Teorema 3.13, vamos inicialmente considerar uma partição $\mathcal{V}$ qualquer de T. Se essa partição for $\gamma$-regular, não há nada a fazer. Caso contrário, usaremos os subconjuntos que testemunham a existência de pares irregulares para subdividir cada parte de $\mathcal{V}$. A partição resultante desse processo será, em um certo sentido, significantemente mais "desordenada" (em termos da variância da densidade entre partes) que $\mathcal{V}$. Argumentaremos, que há um limite para o quão "desordenada" uma partição pode ser. Isso implica que não podemos repetir esse processo indefinidamente, isto é, que eventualmente uma partição regular deverá ser obtida após aplicarmos um certo número de subdivisões.

É preciso introduzir novas definições para que possamos formalizar tais idéias.

Definição 3.14 (refina). Sejam $\mathcal{V}$ e $\mathcal{V}^{\prime}$ partições de um conjunto $V$. Dizemos que $\mathcal{V}^{\prime}$ refina $\mathcal{V}$ se toda parte de $\mathcal{V}^{\prime}$ está inteiramente contida em alguma parte de $\mathcal{V}$.

Definição 3.15 (índice). Sejam $U, W \subset V$ dois conjuntos disjuntos de vértices de um torneio $T_{n}=(V, A)$. Definimos

$$
q(U, W)=\frac{|U||W|}{n^{2}}\left(\vec{d}(U, W)-\frac{1}{2}\right)^{2} .
$$


Se $\mathcal{U}$ e $\mathcal{W}$ são partições de conjuntos disjuntos de $V$, definimos

$$
q(\mathcal{U}, \mathcal{W})=\sum_{\substack{U \in \mathcal{U} \\ W \in \mathcal{W}}} q(U, W)
$$

Seja $\mathcal{V}=\left\{V_{0}, V_{1}, \ldots, V_{m}\right\}$ uma partição de $V$, em que $V_{0}$ é uma parte excepcional. O índice $q(\mathcal{V})$ de $\mathcal{V}$ é dado por $q(\mathcal{V})=\sum q\left(V_{i}, V_{j}\right)$, onde a soma percorre todos os $\left(\begin{array}{c}m+\left|V_{0}\right| \\ 2\end{array}\right)$ pares não ordenados $V_{i}, V_{j}$ de $\mathcal{V}$ - lembrando que estamos considerando aqui que $V_{0}$ é na verdade uma coleção de $V_{0}$ partes unitárias (Notação 3.12).

O índice de uma partição é essencialmente a soma do quadrado das densidades entre os pares de partes. Essa densidade é escalada para levar em conta o tamanho das partes envolvidas. Diferentemente da definição usual para o caso de grafos ou digrafos, optamos por adicionar um fator aditivo $-\frac{1}{2}$ à densidade. Isso torna a definição de $q(U, W)$ simétrica, isto é, $q(U, W)=q(W, U)$. Assim, não precisamos nos preocupar em estabelecer uma ordem para as partes de $\mathcal{V}$ (o que resultaria em outras dificuldades, tais como exigir que partições que refinam $\mathcal{V}$ mantenham essa mesma ordem).

O resultado abaixo mostra que o índice de uma partição está relacionado com a variância das densidades entre as partes que a compõe.

Proposição 3.16. Sejam $\mathcal{U}=\left\{U_{1}, U_{2}, \ldots, U_{k}\right\}$ e $\mathcal{W}=\left\{W_{1}, W_{2}, \ldots, W_{l}\right\}$ partições de conjuntos disjuntos $U$ e $W$ (respectivamente) de vértices de um torneio $T_{n}$. Considere um experimento em que escolhemos uniformemente ao acaso um vértice $u \in U$ e $w \in W$. Defina a variável aleatória $Z:=\vec{d}\left(U_{i}, W_{j}\right)-\frac{1}{2}$, onde $U_{i} \in \mathcal{U}$ e $W_{j} \in \mathcal{W}$ são as partes que contêm $u$ e $w$, respectivamente. Então

$$
\operatorname{Var}(Z)=\frac{n^{2}}{|U||W|}(q(\mathcal{U}, \mathcal{W})-q(U, W))
$$

Demonstração. A esperança de $Z$ é dada por

$$
\begin{aligned}
\mathbb{E}(Z) & =\sum_{\substack{1 \leq i \leq k \\
1 \leq j \leq l}} \frac{\left|U_{i}\right|\left|W_{j}\right|}{|U||W|}\left(\vec{d}\left(U_{i}, W_{j}\right)-\frac{1}{2}\right) \\
& =-\frac{1}{2}+\sum_{\substack{1 \leq i \leq k \\
1 \leq j \leq l}}\left(\frac{\left|U_{i}\right|\left|W_{j}\right|}{|U||W|} \vec{d}\left(U_{i}, W_{j}\right)\right) \\
& =-\frac{1}{2}+\frac{1}{|U||W|} \sum_{\substack{1 \leq i \leq k \\
1 \leq j \leq l}} \vec{e}\left(U_{i}, W_{j}\right) \\
& =\vec{d}(U, W)-\frac{1}{2}
\end{aligned}
$$

e, portanto, $(\mathbb{E}(Z))^{2}=\left(n^{2} /|U||W|\right) q(U, W)$. Concluímos a demonstração observando que

$$
\mathbb{E}\left(Z^{2}\right)=\sum_{\substack{1 \leq i \leq k \\ 1 \leq j \leq l}}\left(\vec{d}\left(U_{i}, W_{j}\right)-\frac{1}{2}\right)^{2}=\sum_{\substack{1 \leq i \leq k \\ 1 \leq j \leq l}} \frac{n^{2}}{\left|U_{i}\right|\left|W_{j}\right|} q\left(U_{i}, W_{j}\right)=\frac{n^{2}}{|U||W|} q(\mathcal{U}, \mathcal{W}) .
$$

Usaremos a Proposição 3.16 para mostrar como subdividir pares de conjuntos irregulares $(U, W)$ de forma a aumentar o índice $q$ associado. 
Lema 3.17. Considere um par $\gamma$-irregular $(U, W)$ de $T_{n}$. Então existem partições $\mathcal{U}=\left\{U_{1}, U_{2}\right\}$ e $\mathcal{W}=\left\{W_{1}, W_{2}\right\}$ tais que

$$
q(\mathcal{U}, \mathcal{W})>q(U, W)+\gamma^{4} \frac{|U||W|}{n^{2}}
$$

Demonstração. Como $(U, W)$ não é $\gamma$-regular, existem $U_{1} \subset U$ e $W_{1} \subset W,\left|U_{1}\right| \geq \gamma|U|$ e $\left|W_{1}\right| \geq$ $\gamma|W|$ tais que $\vec{d}\left(U_{1}, W_{1}\right)$ desvia de $\vec{d}(U, W)$ por mais que $\gamma$. Isso implica que a variável $Z$ (da Proposição 3.16) desvia de $\mathbb{E}(Z)$ por mais que $\gamma$ quando $u \in U_{1}$ e $w \in W_{1}$, ou seja, com probabilidade pelo menos $\gamma^{2}$. Pela desigualdade de Chebyshev (Teorema A.1), concluímos que $\operatorname{Var}(Z)>\gamma^{4}$, da onde segue o resultado desejado pela Proposição 3.16 .

A Proposição 3.16 também nos permite mostrar que ao refinarmos uma partição não diminuímos seu índice.

Lema 3.18. Sejam $\mathcal{V}^{\prime}$ e $\mathcal{V}$ partições de $V$ tais que $\mathcal{V}^{\prime}$ refina $\mathcal{V}$. Então $q\left(\mathcal{V}^{\prime}\right) \geq q(\mathcal{V})$

Demonstração. Primeiro, note que se $\mathcal{U}$ e $\mathcal{W}$ são partições de subconjuntos disjuntos $U, W \subset V$, então devemos ter $q(\mathcal{U}, \mathcal{W}) \geq q(U, W)$ (isso segue diretamente da Proposição 3.16 e do fato da variância ser sempre um valor não negativo). Então sejam $\mathcal{V}=\left\{U_{1}, \ldots, U_{k}\right\}$ e $\mathcal{V}^{\prime}=\bigcup_{i=1}^{k} \mathcal{U}_{i}$, onde cada $\mathcal{U}_{i}$ é uma partição de $U_{i}$. Temos

$$
\begin{aligned}
q\left(\mathcal{V}^{\prime}\right) & =\sum_{A, B \in \mathcal{V}^{\prime}} q(A, B) \\
& =\sum_{1 \leq i<j \leq k} q\left(\mathcal{U}_{i}, \mathcal{U}_{j}\right)+\sum_{1 \leq i \leq k} \sum_{A, B \in \mathcal{U}_{i}} q(A, B) \\
& \geq \sum_{1 \leq i<j \leq k} q\left(\mathcal{U}_{i}, \mathcal{U}_{j}\right) \\
& \geq \sum_{1 \leq i<j \leq k} q\left(U_{i}, U_{j}\right) \\
& =q(\mathcal{V}) .
\end{aligned}
$$

Estamos prontos para mostrar que dada uma partição que não é $\gamma$-regular, podemos obter um refinamento cujo índice supera consideravelmente o da partição original.

Lema 3.19. Seja $\mathcal{V}=\left\{V_{0}, V_{1}, \ldots, V_{m}\right\}$ uma partição de $V$ com uma parte excepcional $V_{0},\left|V_{0}\right| \leq$ $\gamma|V|$, e tal que $\left|V_{1}\right|=\cdots=\left|V_{m}\right|$ têm todos o mesmo tamanho. Suponha que mais do que $\gamma m^{2}$ dos pares $\left(V_{i}, V_{j}\right)$ sejam $\gamma$-irregulares $(1 \leq i<j \leq m)$. Então existe uma partição $\mathcal{V}^{\prime}=\left\{V_{0}^{\prime}, V_{1}^{\prime}, \ldots, V_{m^{\prime}}^{\prime}\right\}$ que refina $\mathcal{V}$ e satisfaz as seguintes propriedades:

(i) Todos os $V_{1}^{\prime}, \ldots V_{m^{\prime}}^{\prime}$ têm o mesmo tamanho.

(ii) $m^{\prime}<m 8^{m}$.

(iii) $\left|V_{0}^{\prime}\right| \leq\left|V_{0}\right|+\frac{n}{2^{m}}$

(iv) $q\left(\mathcal{V}^{\prime}\right) \geq q(\mathcal{V})+\frac{1}{2} \gamma^{5}$ 
Demonstração. Seja $s=\left|V_{1}\right|=\cdots=\left|V_{m}\right|$. Dado um par $\gamma$-irregular $\left(V_{i}, V_{j}\right)$, considere as partições $\mathcal{U}_{i}^{j}$ e $\mathcal{U}_{j}^{i}$ de $V_{i}$ e $V_{j}$ (respectivamente) dadas pelo Lema 3.17. Defina, agora, $\mathcal{W}$ como sendo a partição de menor tamanho que refina $\mathcal{V}$ e cada um $\operatorname{dos} \mathcal{U}_{i}^{j}$ e $\mathcal{U}_{j}^{i}$, para todo par $\gamma$-irregular $\left(V_{i}, V_{j}\right)$. Pelo Lema 3.17 temos

$$
\begin{aligned}
q(\mathcal{W}) & \geq q(\mathcal{V})+\gamma m^{2}\left(\gamma^{4} \frac{s^{2}}{n^{2}}\right) \\
& =q(\mathcal{V})+\gamma^{5}\left(\frac{m s}{n}\right)^{2} \\
& \geq q(\mathcal{V})+\frac{\gamma^{5}}{2} .
\end{aligned}
$$

Para obter a última desigualdade, assumimos ${ }^{3}$ que $\gamma \leq 1-\sqrt{2} / 2$. Infelizmente, a partição $\mathcal{W}$ pode ter conjuntos de tamanho diferentes. Construa então uma partição $\mathcal{V}^{\prime}$, que refina $\mathcal{W}$, subdividindo cada parte de $\mathcal{W}$ arbitrariamente em conjuntos de tamanho $\left\lfloor s / 4^{m}\right\rfloor$, e colocando os vértices que sobram na partição excepcional $V_{0}^{\prime}$. Pelo Lema 3.18, o índice de $\mathcal{V}^{\prime}$ supera o de $\mathcal{V}$ em pelo menos $\frac{1}{2} \gamma^{5}$ — note como adicionar vértices à parte excepcional é uma operação de refinamento.

Agora, observe que para obtermos $\mathcal{W}$ subdividimos cada parte de $\mathcal{V}$ em no máximo $2^{m-1}$ partes. Para obter $\mathcal{V}^{\prime}$, subdividimos cada parte de $\mathcal{W}$ em no máximo $4^{m}$ partes. O total de partes não excepcionais de $\mathcal{V}^{\prime}$ é, portanto, no máximo $m 2^{m-1} 4^{m}<8^{m}$. Ademais, ao construirmos $\mathcal{V}^{\prime}$ adicionamos, para cada parte de $\mathcal{W}$, no máximo $\left\lfloor s / 4^{m}\right\rfloor$ vértices à parte excepcional, de modo que

$$
\left|V_{0}^{\prime}\right| \leq\left|V_{0}\right|+\frac{s}{4^{m}} m 2^{m-1}<\left|V_{0}\right|+\frac{n}{2^{m}}
$$

Antes de prosseguirmos para a demonstração do Teorema 3.13, notamos que o índice de uma partição é limitado superiormente por um valor constante.

Proposição 3.20. Para qualquer partição $\mathcal{V}$ de um torneio, $q(\mathcal{V}) \leq \frac{1}{8}$.

Demonstração. Como para quaisquer $U, V \in \mathcal{V},\left(\vec{d}(U, V)-\frac{1}{2}\right)^{2} \leq \frac{1}{4}$, temos

$$
q(\mathcal{V}) \leq \sum_{U, V \in \mathcal{V}} \frac{|U||V|}{n^{2}} \frac{1}{4} \leq \frac{1}{8 n^{2}}\left(\sum_{U \in \mathcal{V}}|U|\right)\left(\sum_{V \in \mathcal{V}}|V|\right)=\frac{1}{8}
$$

Demonstração do Teorema 3.13. Vamos mostrar que podemos tomar $M=f^{(l)}\left(\mu^{\prime}\right)$, onde $f$ : $\mathbb{N} \rightarrow \mathbb{N}$ é dada por $f(x)=x 2^{x}, \mu^{\prime}:=\max \left\{\mu, \log _{2}\left(\gamma^{-2}\right)-1\right\}$ e $l:=\lceil 1 / 4 \gamma\rceil$.

Considere uma partição arbitrária de $T_{n}$ em $\mu^{\prime}$ partes - cada uma com exatamente $\left\lfloor n / \mu^{\prime}\right\rfloor$ vértices - acrescidas de uma parte excepcional contendo os $\left(<\mu^{\prime}\right)$ vértices restantes. Enquanto essa partição não for $\gamma$-regular, refine-a por meio de sucessivas aplicações do Lema 3.19. Uma partição $\gamma$-regular $\mathcal{V}$ será obtida depois de no máximo $\lceil 1 /(4 \gamma)\rceil$ iterações, caso contrário uma nova aplicação do Lema 3.19 produziria uma partição com índice maior que $\frac{1}{8}$, contradizendo a Proposição 3.20.

O tamanho da parte excepcional $V_{0}$ de $V$ é dado por

$$
\left|V_{0}\right| \leq \mu^{\prime}+\frac{n}{2^{\mu^{\prime}}} \frac{1}{4 \gamma} \leq \mu^{\prime}+\frac{\gamma}{2} n \leq \gamma n
$$

onde a última desigualdade segue de $n \geq M \geq 2 \mu^{\prime} / \gamma$.

\footnotetext{
${ }^{3}$ não há perda de generalidade tendo em vista que, para $\gamma^{\prime}<\gamma$, partições $\gamma^{\prime}$-regulares também são $\gamma$-regulares.
} 
Observação 3.21. Note que, ao aplicar o Teorema 3.13, podemos exigir que a partição regular obtida não só contenha pelo menos $\mu$ partes não excepcionais, mas também que ela refine uma dada partição $\mathcal{U}=\left\{U_{0}, U_{1}, \ldots, U_{\mu}\right\} \operatorname{com}\left|U_{1}\right|=\cdots=\left|U_{\mu}\right|$ e $\left|U_{0}\right|<\mu$.

Ao definirmos uma partição $\gamma$-regular, forçamos a existência de uma parte excepcional apenas para facilitar a demonstração do Lema de Regularidade — essa parte é particularmente útil na demonstração do Lema 3.19, pois ela pode abrigar os vértices que sobram das subdivisões exatas, sem que isso tenha qualquer impacto sobre a índice da partição. Mas podemos reformular o Teorema 3.13 de modo que essa parte excepcional não seja necessária. Nessa reformulação, não podemos mais exigir que as partes tenham todas o mesmo tamanho (por questões de divisibilidade) mas podemos exigir que as cardinalidades delas distem no máximo uma unidade.

Definição 3.22 (equipartição). Uma equipartição $\mathcal{V}=\left\{V_{1}, \ldots, V_{m}\right\}$ de um conjunto $V$ é uma partição de $V$ tal que para todo $1 \leq i<j \leq m,\left|V_{i}\right|=\left|V_{j}\right| \pm 1$.

Definição 3.23 (equipartição $\gamma$-regular). Uma equipartição $\gamma$-regular de um torneio $T=(V, A)$ é uma equipartição $\mathcal{V}=\left\{V_{1}, \ldots, V_{m}\right\}$ de $V$ tal que todos a menos de $\gamma m^{2}$ dos pares $\left(V_{i}, V_{j}\right)$ são $\gamma$-regulares, para $1 \leq i<j \leq m$.

Proposição 3.24. Sejam $X, Y$ conjuntos disjuntos de vértices de um torneio e sejam $\tilde{X} \subseteq X$ e $\tilde{Y} \subseteq Y$ subconjuntos satisfazendo $|\tilde{X}| \leq \gamma^{2}|X|$ e $|\tilde{Y}| \leq \gamma^{2}|Y|$, para alguma constante $0<\gamma<\frac{1}{3}$. Se $(X \backslash \tilde{X}, Y \backslash \tilde{Y})$ é $\gamma$-regular, então $(X, Y)$ é $3 \gamma$-regular.

Demonstração. Sejam $A, B$ conjuntos disjuntos e $\tilde{A} \subseteq A, \tilde{B} \subseteq B$ subconjuntos satisfazendo $|\tilde{A}| \leq$ $p|A|$ e $|\tilde{B}| \leq p|B|$, para alguma constante $0<p<\frac{1}{3}$. Então temos que

$$
q^{2} \vec{d}(A \backslash \tilde{A}, B \backslash \tilde{B}) \leq \vec{d}(A, B) \leq q^{2} \vec{d}(A \backslash \tilde{A}, B \backslash \tilde{B})+2 p q+p^{2}
$$

onde $q=1-p$. Em particular, vale que $\vec{d}(A, B)=\vec{d}(A \backslash \tilde{A}, B \backslash \tilde{B}) \pm 3 p$.

Seja $X^{\prime} \subseteq X$ e $Y^{\prime} \subseteq Y$ tais que $\left|X^{\prime}\right| \geq 3 \gamma|X|$ e $\left|Y^{\prime}\right| \geq 3 \gamma|Y|$. Da afirmação do parágrafo acima, segue que

$$
\begin{aligned}
\vec{d}\left(X^{\prime}, Y^{\prime}\right) & =\vec{d}\left(X^{\prime} \backslash \tilde{X}, Y^{\prime} \backslash \tilde{Y}\right) \pm 3 \frac{\gamma}{3} \\
& =\vec{d}(X \backslash \tilde{X}, Y \backslash \tilde{Y}) \pm 2 \gamma \\
& =\vec{d}(X, Y) \pm\left(2 \gamma+3 \gamma^{2}\right) \\
& =\vec{d}(X, Y) \pm 3 \gamma
\end{aligned}
$$

o que implica que $(X, Y)$ é $3 \gamma$-regular.

Teorema 3.25 ([7] $]_{g r}$ Lema de Regularidade [30] $\left.]_{g r}\right)$. Para todo $0<\gamma<1$ e inteiro $\mu$, existe um inteiro $M=M_{3.25}(\gamma, \mu)$ tal que todo torneio $T_{n}$ com $n \geq M$ admite uma equipartição $\gamma$-regular $\left\{V_{1}, \ldots, V_{m}\right\} \operatorname{com} \mu \leq m \leq M$. 
Demonstração. Seja $\gamma^{\prime}=\frac{1}{10} \gamma^{2}$. Vamos mostrar que podemos tomar $M=M_{3.13}\left(\gamma^{\prime}, \mu\right)$. Aplique o Teorema 3.13 para obter uma partição $\gamma^{\prime}$-regular $\mathcal{U}=\left\{U_{0}, U_{1}, \ldots, U_{m}\right\}$ de $T_{n}$, com $\mu \leq m \leq M$. Particione $U_{0}$ arbitrariamente em $m$ conjuntos $\tilde{V}_{1}, \ldots, \tilde{V}_{m}$, todos de tamanho $\left\lceil\left|U_{0}\right| / m\right\rceil$ ou $\left\lfloor\left|V_{0}\right| / m\right\rfloor$, e considere a equipartição $\mathcal{V}=\left\{U_{i} \sqcup \tilde{V}_{i}\right\}_{1 \leq i \leq m}$ de $T_{n}$.

Para todo $1 \leq i \leq m$, temos

$$
\frac{\left|\tilde{V}_{i}\right|}{\left|V_{i}\right|} \leq \frac{\left\lceil\frac{\gamma^{\prime} n}{m}\right\rceil}{\left\lfloor\frac{n}{m}\right\rfloor} \leq\left(\frac{\gamma}{3}\right)^{2}
$$

para $n$ suficientemente grande. Como todos a menos de $\epsilon m^{2}$ dos pares $\left(U_{i}, U_{j}\right)(1 \leq i<j \leq m)$ são, em particular, $\frac{1}{3} \gamma$-regulares, a Proposição 3.24 implica $\mathcal{V}$ ser uma partição $\gamma$-regular de $\mathcal{V}$.

Observação 3.26. Também podemos exigir que a equipartição regular obtida a partir do teorema acima refine uma dada partição de tamanho $\mu$ (ver Observação 3.21).

\subsubsection{Considerações sobre o Lema}

A demonstração do Lema de Regularidade garante apenas que o tamanho máximo $M$ da partição dada pelo lema é limitado por TOWER $\left(\left\lceil\gamma^{-5}\right\rceil\right)$. Por outro lado, Gowers [24] mostrou que existem exemplos (de grafos) para os quais toda partição $\gamma$-regular deve conter pelo menos TOWER( $\left.\operatorname{poli}\left(\gamma^{-1}\right)\right)$, isto é, que essa cota superior para $M$ não pode ser melhorada significativamente.

\subsection{Imersão de torneios em pares regulares}

Nesta seção veremos como o Lema de Regularidade pode ser usado para demonstrar o Teorema 3.5. Suponha que queiramos encontrar muitas cópias de um torneio $T_{h}=\left(\left\{u_{1}, \ldots, u_{h}\right\}, A_{h}\right)$ em um torneio $T=(V, A)$. Vamos mostrar que é suficiente encontrar conjuntos disjuntos (de cardinalidade considerável) $U_{1}, \ldots, U_{h}$ de vértices de $V$, dois a dois regulares, e que respeitem as mesmas relações de dominância de $T_{h}$, isto é, se $u_{i} \rightarrow u_{j}$, então o par é $\left(U_{i}, U_{j}\right)$ tem alta densidade, para todo $1 \leq i<j \leq h$.

Proposição 3.27. Para qualquer $0<\eta<1$ e inteiro $h$, existem números reais positivos $\gamma=$ $\gamma_{3.27}(\eta, h)$ e $\delta=\delta_{3.27}(\eta, h)$ satisfazendo o seguinte. Sejam $T=(V, A)$ um torneio e $\mathcal{U}$ uma coleção de $h$ subconjuntos de $V$, dois a dois disjuntos e $\gamma$-regulares. Seja $T_{h}$ é um torneio de $h$ vértices admitindo uma função bijetora $\phi: V\left(T_{h}\right) \rightarrow \mathcal{U}$ com a propriedade de que, para qualquer arco $\overrightarrow{v w}$ de $T_{h}$, $\vec{d}(\phi(v), \phi(w)) \geq \eta$. Então existem pelo menos $\delta m^{h}$ cópias de $T_{h}$ em $T$, onde $m=\min \{|U|: U \in \mathcal{U}\} . \diamond$

Demonstração. Por indução em $h$. O resultado é trivial para $h=1$ (tome $\gamma=\delta=1$ ). Suponha então que $h>1$ e que o resultado vale para torneios $T_{h-1}$ e partições $\mathcal{U}^{\prime}$ de tamanho $h-1$. Mostraremos que podemos tomar

$$
\begin{gathered}
\gamma=\gamma_{3.27}(\eta, h)=\min \left\{\frac{1}{2(h-1)}, \frac{\eta}{2}, \gamma_{3.27}\left(\frac{1}{2} \eta, h-1\right) \frac{\eta}{2}\right\} \mathrm{e} \\
\delta_{3.27}(\eta, h)=\frac{1}{2}(\eta-\gamma)^{h-1} \delta_{3.27}(\eta, h-1) .
\end{gathered}
$$

Fixe um vértice $v \in T_{h}$ e seja $W=\phi(v) \in \mathcal{U}$. Defina, ainda,

$$
\mathcal{U}^{+}=\{U \in \mathcal{U} \backslash\{W\}: \vec{d}(W, U) \geq \eta\}
$$


$\mathrm{e}$

$$
\mathcal{U}^{-}=\{U \in \mathcal{U} \backslash\{W\}: \vec{d}(U, W) \geq \eta) .
$$

Pela Proposição 3.10, fixado $U^{+} \in \mathcal{U}^{+}$, no máximo $\gamma m$ vértices de $W$ dominam menos que $(\eta-\gamma) m$ vértices de $U^{+}$. De forma simétrica, no máximo $\gamma m$ vértices de $W$ são dominados por menos que $(\eta-\gamma) m$ vértices de cada $U^{-}$fixo em $\mathcal{U}^{-}$. Então existe um conjunto $W^{\prime} \subseteq W$, de tamanho pelo menos $(1-(h-1) \gamma) m \geq \frac{1}{2} m$, tal que para qualquer $w \in W$, temos $g_{U^{+}}^{+}(w) \geq(\eta-\gamma) m$ e $g_{U^{-}}^{-}(w) \geq(\eta-\gamma) m$, para todo $U^{+} \in \mathcal{U}^{+}$e $U^{-} \in \mathcal{U}^{-}$.

Fixe um vértice $w \in W^{\prime}$ e defina a coleção

$$
\mathcal{U}^{\prime}=\left\{\Gamma_{U^{+}}^{+}(w): U^{+} \in \mathcal{U}^{+}\right\} \cup\left\{\Gamma_{U^{-}}^{-}(w): U^{-} \in \mathcal{U}^{-}\right\}
$$

Note que cada $U^{\prime} \in \mathcal{U}^{\prime}$ tem tamanho pelo menos $(\eta-\gamma) m$. Além disso, como $\gamma \leq \frac{1}{2} \eta$, podemos aplicar a Proposição 3.9, para concluir que todos os pares de conjuntos de $\mathcal{U}^{\prime}$ são $\gamma /(\eta-\gamma) \leq$ $(2 \gamma / \eta) \leq \gamma_{3.27}\left(\frac{1}{2} \eta, h-1\right)$-regulares com densidade pelo menos $\eta-\gamma \geq \frac{1}{2} \eta$.

Estamos finalmente em condições de usar a hipótese de indução sobre o torneio $T_{h-1}:=T_{h}-v$ e a coleção $\mathcal{U}^{\prime}$, e concluir a existência de $\delta_{3.27}\left(\frac{1}{2} \eta, h-1\right)(\eta-\gamma)^{h-1} m^{h-1}$ cópias de $T_{h-1}$ em $T$. Cada uma delas, somada ao vértice $w$, forma uma cópia distinta de $T_{h}$. Repetindo o mesmo argumento para todo $w \in W^{\prime}$, aferimos a existência de pelo menos $\delta m^{h}$ cópias distintas de $T_{h}$ em $T$.

Esse resultado nos mostra que pares regulares se comportam de maneira semelhante a pares onde os arcos são escolhidos de forma aleatória: se o sentido dos arcos entre $U_{i}$ e $U_{j}$ apontassem para $U_{j}$ com probabilidade $\eta$ (independentemente das demais escolhas) sempre que $u_{i} \rightarrow u_{j}$, esperaríamos encontrar, em média, $\prod_{i}\left|U_{i}\right| \eta^{h}$ cópias de $T_{h}$ em $T\left[U_{1}, \ldots U_{h}\right]$ e resultados de concentração nos indicariam que, com alta probabilidade, o número real de cópias não desvia muito desta quantidade.

A Proposição 3.27 pode ser reescrita de forma mais simples, com o auxílio da seguinte definição.

Definição 3.28 (digrafo reduzido). Sejam $T=(V, A)$ um torneio e $\mathcal{U}$ uma coleção de $k$ subconjuntos de $V$, dois a dois disjuntos. Dados reais $0 \leq \eta, \gamma \leq 1$, definimos o digrafo reduzido $R=R_{\eta}^{\gamma}(T, \mathcal{U}):=\left(\mathcal{U}, A_{R}\right)$ como um digrafo (cujos vértices são os elementos de $\mathcal{U}$ ) satisfazendo $U \stackrel{R}{\rightarrow} U^{\prime}$ se, e somente se, $\left(U, U^{\prime}\right)$ é $\gamma$-regular e $\vec{d}\left(U, U^{\prime}\right) \geq \eta$ (note que o digrafo reduzido pode conter laços).

Lema 3.29. Para qualquer $0<\eta<1$ e inteiros positivos $h$ e $M$, existem constantes positivas $\gamma=\gamma_{3.29}(\eta, h, M)$ e $\delta=\delta_{3.29}(\eta, h, M)$ satisfazendo o seguinte. Sejam $T=(V, A)$ um torneio e $\mathcal{V}$ uma equipartição de $T$ de cardinalidade no máximo $M$. Então qualquer torneio $T_{h}$ que seja subgrafo de $R_{\eta}^{\gamma}(T, \mathcal{V})$ é $\delta$-abundante em $T$.

Demonstração. Basta aplicar a Proposição 3.27, usando $\phi$ como a função que atesta que $T_{h}$ é subgrafo de $R_{\eta}^{\gamma}(T, \mathcal{V})$.

Demonstração do Teorema 3.5. Seja $T_{h}$ um torneio $\epsilon$-inevitável em $T_{n}=(V, A)$ e defina $\gamma:=$ $\min \left\{\frac{1}{3} \epsilon, \gamma_{3.27}\left(\frac{1}{3} \epsilon, h\right)\right\}$. Aplique o Teorema 3.25 sobre $T_{n}$ para obter uma equipartição $\gamma$-regular de $\mathcal{V}=\left\{V_{1}, \ldots, V_{m}\right\}$ com $\frac{3}{\epsilon} \leq m \leq M:=M_{3.25}\left(\gamma, \frac{3}{\epsilon}\right)$.

Considere o conjunto $S \subseteq A$ formado pela união dos seguintes subconjuntos de $\operatorname{arcos}$ de $T$ :

- O conjunto $S_{1}$ de arcos internos a cada parte $V_{i} \in \mathcal{V}$. Note que $\left|S_{1}\right|<m\left(\frac{n}{m}\right)^{2}=n^{2} / m \leq \frac{1}{3} \epsilon n^{2}$. 
- O conjunto $S_{2}$ dos arcos que cruzam pares $\left(V_{i}, V_{j}\right)$ irregulares. Note que $\left|S_{2}\right| \leq \gamma m^{2} \frac{n}{m} \frac{n}{m} \leq$ $\frac{1}{3} \epsilon n^{2}$.

- O conjunto $S_{3}$ de arcos que cruzam pares $\left(V_{i}, V_{j}\right)$ tais que $\vec{d}\left(V_{i}, V_{j}\right)<\frac{1}{3} \epsilon$ ou $\vec{d}\left(V_{j}, V_{i}\right)<\frac{1}{3} \epsilon$. Note que $S_{3} \mid<m^{2} \frac{1}{3} \epsilon \frac{n}{m} \frac{n}{m} \leq \frac{1}{3} \epsilon n^{2}$.

Como $S$ tem cardinalidade menor que $\epsilon n^{2}$, deve existir alguma cópia de $T_{h}$ que não contém arcos pertencentes a $S$. Considere a função em $V\left(T_{h}\right) \rightarrow \mathcal{V}$ que leva cada vértice dessa cópia à parte $V \in \mathcal{V}$ a qual ele pertence. Essa função atesta que $T_{h}$ é subgrafo de $R_{\eta / 3}^{\gamma}(T, \mathcal{V})$.

Concluímos a demonstração aplicando o Lema 3.29 para concluir que $T_{h}$ é $\delta_{3.29}(\eta / 3, h, M)$ abundante em $T$. 


\section{Capítulo 4}

\section{Testando subtorneios}

O principal objetivo deste capítulo consiste em mostrar o seguinte resultado.

Teorema $4.1\left([3]_{g r}\right)$. A propriedade Livre $\left(T_{h}\right)$ é testável com erro-unilateral.

O resultado combinatório necessário para provar tal teorema consiste em mostrar que torneios longe de serem livres de $T_{h}$ contêm muitas cópias de $T_{h}$.

Teorema 4.2 (Lema de Remoção para Torneios $\left.[3]_{g r}\right)$. Seja $T_{h}$ um torneio com $h$ vértices. Para todo inteiro $h$ e $0<\epsilon<1$, existe $\delta=\delta_{4.2}(\epsilon, h)$ tal que se $T$ é um torneio $\epsilon$-longe de ser livre de $T_{h}$, então $T_{h}$ é $\delta$-abundante em $T$.

Tendo em mãos o Teorema 4.2, é fácil construir um testador com erro-unilateral para Livre $\left(T_{h}\right)$. Basta amostrar um conjunto $Q$ de vértices de $T$, de cardinalidade suficientemente grande, e verificar se há alguma cópia de $T_{h}$ em $T[Q]$.

Demonstração do Teorema 4.1. Dado $\epsilon$ e um torneio $T=(V, A)$ de entrada, considere um testador para Livre $\left(T_{h}\right)$ que procede da seguinte forma. Primeiramente amostramos, uniforme e independentemente ao acaso, $\left\lceil 2 \delta^{-1}\right\rceil h$ vértices de $V$ e consideramos o conjunto $Q$ formado por tais vértices ${ }^{1}$. Em seguida aceitamos $T$ se, e somente se, $T[Q]$ é livre de $T_{h}$.

É evidente que se $T \in \operatorname{Livre}\left(T_{h}\right)$, então $T[Q] \in \operatorname{Livre}\left(T_{h}\right)$ e, portanto, o algoritmo o aceita com probabilidade 1 . Suponha então que $T$ está $\epsilon$-longe de Livre $\left(T_{h}\right)$. Precisamos provar que, nesse caso, $\mathbb{P}\left(T_{n}\right.$ ser aceito $)<\frac{1}{3}$. Pelo Teorema 4.2, a probabilidade de uma amostra de tamanho $h$ formar uma cópia de $T_{h}$ é pelo menos $\delta$. Como o conjunto $Q$ possui $\left\lceil 2 \delta^{-1}\right\rceil h$-uplas distintas, concluímos que

$$
\mathbb{P}(T \text { ser aceito })<(1-\delta)^{2 \delta^{-1}}=\left(1-\frac{1}{\delta^{-1}}\right)^{2 \delta^{-1}} \leq e^{-2}<\frac{1}{3},
$$

como desejado.

Para encontrar muitas cópias de um torneio $T_{h}$ em $T$, uma idéia natural seria fazer uso da Proposição 3.27. Gostaríamos de poder proceder de forma similar à feita para torneios nos quais $T_{h}$ é inevitável, isto é, tomar uma partição regular de um torneio longe de Livre $\left(T_{h}\right)$ e argumentar que existe uma cópia de $T_{h}$ que atesta a existência de conjuntos como nas condições da Proposição 3.27.

\footnotetext{
${ }^{1}$ estamos assumindo (ver Observação 2.25) que tais vértices são distintos.
} 
Tal tarefa era mais simples naquele caso, pois podíamos simplesmente eleger arcos que não podiam ser usados para formar cópias de $T_{h}$. Agora, nossa única arma consiste em inverter arcos.

Certamente, podemos reorientar arcos entre partes regulares de forma a evitar que os arcos das cópias de $T_{h}$ (que sobrevivem a tais alterações) cruzem partes cuja densidade é baixa naquele sentido. Por outro lado, não é imediatamente claro como evitar que

(i) arcos internos a uma mesma parte sejam usados para formar cópias de $T_{h} \mathrm{e}$

(ii) arcos entre partes irregulares sejam usados para formar cópias de $T_{h}$.

O primeiro problema tem solução relativamente simples, que será apresentada na próxima seção. O segundo exigirá uma versão mais forte do Lema de Regularidade, que nos permita evitar pares irregulares de alguma forma.

\subsection{Abundância de um subtorneio é fechada por homomorfismo}

A Proposição 3.27, aplicada indiretamente para demonstrar o Teorema 3.5, garante que um subtorneio $T_{h}$ é abundante em um torneio $T$ quando há uma função injetora que leva vértices de $T_{h}$ à partes $U_{1}, \ldots U_{k}$ duas a duas regulares de $T$ e com densidades apropriadas. Nesta seção, mostraremos como enfraquecer as condições da Proposição 3.27, de modo que essa função não precise ser necessariamente injetora. Mais precisamente, permitiremos que tal função seja um homomorfismo.

O seguinte resultado é uma consequência imediata do Teorema 2.12.

Proposição 4.3. Para todo inteiro $k>0$ existe uma constante $\alpha=\alpha(k)$ tal que o torneio transitivo $\pi_{k}$ é $\alpha$-abundante em qualquer torneio $T_{n}$ (suficientemente grande).

Demonstração. É suficiente mostrar que há pelo menos $\alpha n^{k}$ cópias não rotuladas de $\pi_{k}$ em $T_{n}$. Como cada conjunto de tamanho $R:=N_{2.12}(k)$ induz pelo menos uma cópia não rotulada de $\pi_{k}$, o número de tais cópias é pelo menos

$$
\left(\begin{array}{l}
n \\
R
\end{array}\right)\left(\begin{array}{l}
n-k \\
R-k
\end{array}\right)^{-1} \geq\left(\frac{n}{R}\right)^{R}\left(\frac{R-k}{e n}\right)^{R-k}=\alpha n^{k},
$$

onde

$$
\alpha=\frac{(R-k)^{R-k}}{R^{R} e^{R-k}} .
$$

A seguinte operação nos permitirá trabalhar com homomorfismos de forma mais controlada.

Definição 4.4 (expansão). Sejam $T=(V, A)$ um torneio, $v \in V$ e $r$ um inteiro positivo. Definimos o torneio $\mathcal{B}_{r}(T, v)$, resultante da operação de expansão sobre $v$, como uma cópia de $T$ em que substituímos $v$ por um torneio transitivo de tamanho $r$. Mais especificamente, definimos

$$
V\left(\mathcal{B}_{r}(T, v)\right)=V \backslash\{v\} \sqcup\left\{u_{1}, \ldots, u_{r}\right\}
$$

e

$$
\begin{aligned}
A\left(\mathcal{B}_{r}(T, v)\right)=A \backslash\{\overrightarrow{x v}: x \in V\} \backslash\{\overrightarrow{v x}: x \in V\} & \cup\left\{\overrightarrow{u_{i} u_{j}}: 1 \leq i<j \leq r\right\} \\
& \cup\left\{\overrightarrow{u_{i} x}: 1 \leq i \leq r \text { e } v \in V \text { tal que } v \stackrel{T}{\rightarrow} x\right\} \\
& \cup\left\{\overrightarrow{x u_{i}}: 1 \leq i \leq r \text { e } v \in V \text { tal que } x \stackrel{T}{\rightarrow} v\right\} .
\end{aligned}
$$


O próximo resultado afirma que torneios que têm muitas cópias de um torneio $T_{h}$ também contêm muitas cópias de torneios obtidos por operações de expansão sobre $T_{h}$.

Lema 4.5. Para todo inteiro positivo $r$ e número real $\delta>0$, existe uma constante positiva $\delta_{\mathcal{B}}=$ $\delta_{\mathcal{B}}(\delta, r)=\operatorname{poli}_{<}(\delta)$ tal que se um torneio $T_{h}$ é $\delta$-abundante em um torneio $T_{n}$, então $\mathcal{B}_{r}\left(T_{h}, v\right)$ é $\delta_{\mathcal{B}}$-abundante em $T_{n}$, para qualquer $v \in V\left(T_{h}\right)$.

Demonstração. Seja $T_{h-1}=T_{h}-v$ o torneio obtido de $T_{h}$ após a remoção do vértice $v$ e dos arcos incidentes a $v$. Sejam $W_{1}, \ldots, W_{N}$ todas as $(h-1)$-uplas que formam cópias de $T_{h-1}$ em $T_{n}$. Note que devemos ter $\delta n^{h-1}<N<n^{h-1}$. Para cada $W_{i}$ fixo, definimos $d\left(W_{i}\right)$ como o número de vértices que, junto com $W_{i}$, formam uma cópia de $T_{h}$ (em particular, devemos ter $\sum_{i=1}^{N} d\left(W_{i}\right)=\delta n^{h}$ ).

Seja $\alpha=\alpha_{4.3}(r)$. Segue da Proposição 4.3 que o número de cópias de $\mathcal{B}_{r}\left(T_{h}, v\right)$ em $T_{n}$ é maior ou igual a

$$
\sum_{i=1}^{N} \alpha d\left(W_{i}\right)^{r} \geq N \alpha\left(\frac{\sum_{i=1}^{N} d\left(W_{i}\right)}{N}\right)^{r} \geq \alpha \delta n^{h-1}\left(\frac{\delta n^{h}}{n^{h-1}}\right)^{r}=\underbrace{\alpha \delta^{r+1}}_{\delta_{\mathcal{B}}} n^{h+r-1},
$$

onde a primeira desigualdade acima segue da convexidade da função $z \mapsto \alpha z^{r}$.

Com posse do resultado acima, estamos prontos para demonstrar uma versão mais forte da Proposição 3.27 .

Lema 4.6 (imersão de torneios). Para quaisquer $0<\eta<1,0<\lambda<1$, e inteiros $h$ e $k$, existem números reais positivos $\gamma=\gamma_{4.6}(\eta, h)$ e $\delta=\delta_{4.6}(\eta, \lambda, h, k)$ satisfazendo o seguinte.

Seja $T=(V, A)$ um torneio e $\mathcal{U}=\left\{U_{1}, \ldots, U_{k}\right\}$ uma coleção de $k$ subconjuntos de $V$ de cardinalidade pelo menos $\lambda|V|$, dois a dois disjuntos e $\gamma$-regulares. Seja $T_{h}$ um torneio tal que $T_{h} \stackrel{\text { hom }}{\longrightarrow} R_{\eta}(T, \mathcal{U})$. Então $T_{h}$ é $\delta$-abundante em $T$.

Demonstração. Seja $\phi$ um homomorfismo de $T_{h}$ para $R:=R_{\eta}(T, \mathcal{U})$. Considere o torneio $T_{h^{\prime}}=$ $R\left[\phi\left(V\left(T_{h}\right)\right)\right]$ uma enumeração $v_{1}, \ldots, v_{h^{\prime}}$ dos vértices de $T_{h^{\prime}}$. Defina, ainda, os seguintes $h+1$ torneios:

$$
\begin{gathered}
T^{(0)}=T_{h^{\prime}}, \\
T^{(i)}=\mathcal{B}_{\left|\phi^{-1}\left(v_{i}\right)\right|}\left(T^{(i-1)}, v_{i}\right) \text { para } 1 \leq i \leq h^{\prime} .
\end{gathered}
$$

Se tomarmos $\gamma=\gamma_{3.27}\left(\eta, h^{\prime}\right)$, então $T^{(0)}$ e $\mathcal{U}$ satisfazem as condições da Proposição 3.27. Concluímos que $T^{(0)}$ é $\delta^{(0)}$-abundante em $T$, onde $\delta^{(0)}=\delta_{3.27}\left(\eta, h^{\prime}\right) \lambda^{k}$.

Para todo $1 \leq i \leq h^{\prime}$, segue do Lema 4.5 que se $T^{(i-1)}$ é $\delta^{(i-1)}$-abundante em $T$, então existe $\delta^{(i)}>0$ tal que $T^{(i)}$ é $\delta^{(i)}$-abundante em $T$.

Concluímos por indução em $h^{\prime}$ que $T_{h}=T^{\left(h^{\prime}\right)}$ é $\delta^{\left(h^{\prime}\right)}$-abundante em $T$, para algum $\delta^{\left(h^{\prime}\right)}>0$.

\subsection{Lema forte da regularidade}

Para demonstrar o Teorema 4.2, é suficiente argumentar que todo torneio $T_{n}$ que está $\epsilon$-longe de $\operatorname{Livre}\left(T_{h}\right)$ admite uma coleção $\mathcal{U}$ de conjuntos de vértices que satisfaçam as condições do Lema 4.6. A estratégia que usaremos consistirá em considerar uma partição regular de $T$ e inverter a orientação 
de um certo conjunto de arcos (de cardinalidade inferior a $\epsilon n^{2}$ ), de modo que as cópias de $T_{h}$ que sobrevivem a tais alterações atestem a existência de uma tal coleção $\mathcal{U}$.

Como discutido no início deste capítulo, pares irregulares impõe uma dificuldade à essa estratégia. Uma pergunta natural é, portanto, se não é possível derivar uma versão mais forte do Teorema 3.25 que garanta a existência de uma partição em que todos os pares de partes são regulares. Essa pergunta foi feita inicialmente por Szemerédi [30] em relação a grafos. Lovász, Seymour e Trotter e, independentemente, Alon, Duke, Lefman, Rödl e Yuster [1] observaram que pares irregulares são de fato inevitáveis em qualquer equipartição de certos grafos específicos. O exemplo canônico é o chamado half-graph, um grafo bipartido com partes $\left\{a_{1}, \ldots, a_{n}\right\}$ e $\left\{b_{1}, \ldots, b_{n}\right\}$ tal que a aresta $a_{i} b_{j}$ é presente se e só se $i \leq j$ - para este grafo, o número de pares irregulares deve ser linear no número $m$ de partes. Mais recentemente, Conlon e Fox [14] mostraram uma construção em que o número de pares irregulares deve ser pelo menos $\frac{C m^{2}}{\log ^{*} m}$, para alguma constante $C$, o que mostra que o número de pares irregulares fornecido pelo Lema de Regularidade não pode ser substancialmente melhorado.

Apesar de pares irregulares serem inevitáveis em uma partição, foi mostrado em [3] que é possível obter uma partição regular tal que cada parte contém um subconjunto que a "representa" (em termos de densidade) e todos esses subconjuntos são dois a dois regulares.

Teorema 4.7 (Lema Forte da Regularidade [3] $]_{\text {gr }}$ ). Para todo inteiro $\mu$ e função $\mathcal{E}: \mathbb{N} \rightarrow$ $(0 ; 1)$, existe um inteiro $M=M_{4.7}(\mathcal{E}, \mu)$ e uma constante $\lambda=\lambda_{4.7}(\mathcal{E}, \mu)>0$ satisfazendo o seguinte. Para todo torneio $T_{n}, n>M$, existe uma equipartição $\mathcal{V}=\left\{V_{1}, \ldots, V_{m}\right\}$ e conjuntos de vértices $V_{i}^{\prime} \subseteq V_{i}, 1 \leq i \leq m$ tais que:

$-\mu \leq m \leq M$.

$-\left|V_{i}^{\prime}\right| \geq \lambda n$.

- Todos os pares $\left(V_{i}^{\prime}, V_{j}^{\prime}\right)$ são $\mathcal{E}(m)$-regulares, $1 \leq i<j \leq m$.

- Todos a menos de $\mathcal{E}(0) m^{2}$ dos pares $1 \leq i \leq j \leq m$ são tais que $\vec{d}\left(V_{i}, V_{j}\right)=\vec{d}\left(V_{i}^{\prime}, V_{j}^{\prime}\right) \pm \mathcal{E}(0)$.

O teorema acima também fortalece o Lema de Regularidade ao permitir que possamos especificar o quão regular são os pares $\left(V_{i}^{\prime}, V_{j}^{\prime}\right)$ em função do tamanho $m$ da partição. Isso será usado para mostrar que propriedades hereditárias de torneios são testáveis. Neste capítulo, contudo, aplicaremos o Teorema 4.7 usando funções $\mathcal{E}$ constantes.

Lema 4.8. Seja $\mathcal{U}=\left\{V_{i, k}: 1 \leq i \leq m, 1 \leq k \leq l\right\}$ uma equipartição de um torneio $T_{n}$ que refina uma equipartição $\mathcal{V}=\left\{V_{i}: 1 \leq i \leq m\right\}\left(V_{i, k} \subset V_{i}\right)$. Se $n \geq 2 m l$ e $q(\mathcal{U})-q(\mathcal{V}) \leq \frac{1}{16} \epsilon^{4}$, então todos a menos de no máximo $\epsilon m^{2}$ dos pares $1 \leq i<j \leq m$ satisfazem $\vec{d}\left(V_{i, k}, V_{j, k^{\prime}}\right)=\vec{d}\left(V_{i}, V_{j}\right) \pm \epsilon$, para pelo menos $(1-\epsilon) l^{2}$ dos pares $1 \leq k, k^{\prime} \leq l$.

Demonstração. Fixe um par $V_{i}, V_{j} \in \mathcal{V}$. Suponha que mais do que $\epsilon l^{2}$ dos pares $k, k^{\prime}$ sejam tais que $\vec{d}\left(V_{i, k}, V_{j, k^{\prime}}\right)$ desvia de $\vec{d}\left(V_{i}, V_{j}\right)$ por mais que $\epsilon$. Defina $\mathcal{V}_{i}$ e $\mathcal{V}_{j}$ como sendo as partições de $V_{i}$ e $V_{j}$ determinadas por $\mathcal{U}$. 
Seja $Z$ a variável aleatória do enunciado da Proposição 3.16 para as partições $V_{i}$ e $V_{j}$. Segue da desigualdade de Chebyshev (Teorema A.1) que

$$
\operatorname{Var}(Z) \geq \frac{\left\lfloor\frac{\left|V_{i}\right|}{l}\right\rfloor\left\lfloor\frac{\left|V_{j}\right|}{l}\right\rfloor}{\left|V_{i}\right|\left|V_{j}\right|} \epsilon^{3} l^{2} \geq \frac{1}{4} \epsilon^{3} .
$$

Pela Proposição 3.16, concluímos que

$$
q\left(\mathcal{V}_{i}, \mathcal{V}_{j}\right)-q\left(V_{i}, V_{j}\right) \geq \frac{\left|V_{i}\right|\left|V_{j}\right|}{n^{2}} \frac{1}{4} \epsilon^{3} \geq \frac{1}{16 m^{2}} \epsilon^{3} .
$$

Assim, se houver mais do que $\epsilon m^{2}$ pares $V_{i}, V_{j}$ como esses, teremos

$$
q(\mathcal{U})-q(\mathcal{V})=\sum_{i<j} q\left(\mathcal{V}_{i}, \mathcal{V}_{j}\right)-q\left(V_{i}, V_{j}\right) \geq \frac{1}{16} \epsilon^{4}
$$

Lema 4.9. Para todo inteiro $\mu$ e função $\mathcal{E}: \mathbb{N} \rightarrow(0 ; 1)$, existe um inteiro $M=M_{4.9}(\mathcal{E}, \mu)$ tal que todo torneio $T_{n}, n \geq M$ admite uma equipartição $\mathcal{U}=\left\{V_{i, k}: 1 \leq i \leq m, 1 \leq k \leq l\right\}$ que refina uma equipartição $\mathcal{V}=\left\{V_{i}: 1 \leq i \leq m\right\}$ com as seguintes propriedades:

$-m \geq \mu$ e $m l \leq M$

- V é uma equipartição $\mathcal{E}(0)$-regular de $T_{n}$

- Para todo $1 \leq i<j \leq m$, no máximo $\mathcal{E}(m) l^{2}$ dos pares $\left(V_{i, k}, V_{j, k^{\prime}}\right)$ são $\mathcal{E}(m)$-irregulares.

- Todos a menos de no máximo $m^{2}$ dos pares $V_{i}, V_{j} \in \mathcal{V}$ são tais que $\vec{d}\left(V_{i, k}, V_{j, k^{\prime}}\right)=\vec{d}\left(V_{i}, V_{j}\right) \pm \epsilon$ para pelo menos $(1-\epsilon) l^{2}$ dos pares $\left(V_{i, k}, V_{j, k^{\prime}}\right)$.

Demonstração. Seja $M_{1}:=M_{3.25}(\mathcal{E}(0), \mu)$ e para todo $r>1$

$$
M_{r}:=M_{3.25}\left(M_{r-1}, \frac{\mathcal{E}\left(M_{r-1}\right)}{M_{r-1}^{2}}\right) .
$$

Aplique o Teorema 3.25 ao torneio $T_{n}$ para obter uma equipartição $\mathcal{E}(0)$-regular $\mathcal{V}_{1}$ de tamanho no máximo $M_{1}$. Para todo $r>1$, seja $\mathcal{V}_{r}$ uma equipartição $\left(\mathcal{E}\left(M_{r-1}\right) M_{r-1}^{-2}\right)$-regular de $T_{n}$ que refina $\mathcal{V}_{r-1}$ e tem tamanho no máximo $M_{r}\left(\mathcal{V}_{r}\right.$ pode ser obtida aplicando o Teorema 3.25 à partição $\mathcal{V}_{r-1}$ - ver Observação 3.26).

Considere o menor $s>1$ tal que $q\left(V_{s}\right)-q\left(V_{s-1}\right) \leq \frac{1}{16} \epsilon^{4}$ (garantimos a existência de $s$, uma vez que $0 \leq q\left(V_{r-1}\right) \leq q\left(V_{r}\right) \leq \frac{1}{8}$, para todo $r>1$ ). Afirmamos que podemos tomar $\mathcal{V}=\mathcal{V}_{s-1}, \mathcal{U}=\mathcal{V}_{s}$, $m=\left|\mathcal{V}_{s-1}\right|, l=\left|\mathcal{V}_{s}\right|$ e $M=M_{s}$

De fato, é evidente que $\mathcal{V}$ e $\mathcal{U}$ satisfazem as restrições de cardinalidade e $\mathcal{V}$ é $\mathcal{E}(0)$-regular ${ }^{2}$. Como dentre todos os pares de partes de $\mathcal{U}$, no máximo $\frac{\mathcal{E}(m)}{m^{2}}(m l)^{2}=\mathcal{E}(m) l^{2}$ são $\mathcal{E}(m)$-irregulares, a terceira condição é, em particular, satisfeita. Além disso, a última condição do enunciado é satisfeita pelo Lema 4.8 .

Demonstração do Teorema 4.7. Defina $\mathcal{E}(m)=\min \left\{\mathcal{E}(m), \frac{1}{8} \mathcal{E}(0), \frac{1}{4} m^{2}\right\}$ e vamos tomar $M=$ $M_{4.9}(\mathcal{E}, \mu)$ e $\lambda=M^{-1}$. Aplique o Lema 4.9 com parâmetros $\mathcal{E}$ e $\mu$ ao torneio $T_{n}$ para obter equipartições $\left\{V_{i}: 1 \leq i \leq m\right\}$ e $\left\{V_{i, k}: 1 \leq i \leq m, 1 \leq k \leq l\right\}$ satisfazendo as condições do lema.

\footnotetext{
${ }^{2}$ assumindo sem perda de generalidade que $\mathcal{E}$ é decrescente.
} 
Para todo $1 \leq i \leq m$, escolha, uniforme e independentemente ao acaso, inteiros $j(i), 1 \leq j(i) \leq l$ e faça $V_{i}^{\prime}:=V_{i, j(i)}$. Pela cota da união, todos os pares $\left(V_{i}^{\prime}, V_{j}^{\prime}\right)$ são $\mathcal{E}(k)$-regulares com probabilidade pelo menos $\frac{1}{4}$. Ademais, o número médio de pares $i<j$ para os quais $\mid \vec{d}\left(V_{i}, V_{j}-\vec{d}\left(V_{i}^{\prime}, V_{j}^{\prime}\right) \mid>\epsilon\right.$ é limitado superiormente por

$$
\frac{1}{8} \mathcal{E}(0) \frac{m^{2}}{\left(\begin{array}{c}
m \\
2
\end{array}\right)}\left(\begin{array}{c}
m \\
2
\end{array}\right)+\frac{1}{8} \mathcal{E}(0)\left(\begin{array}{c}
m \\
2
\end{array}\right) \leq \frac{1}{4} \mathcal{E}(0) m^{2}
$$

Portanto, o número desses pares é no máximo $\mathcal{E}(0) \mathrm{m}^{2}$, com probabilidade pelo menos $\frac{1}{4}$. Assim, com probabilidade pelo menos $1-\frac{1}{2}-\frac{1}{4}=\frac{1}{4}$, os conjuntos $\left\{V_{i}\right\}_{1 \leq i \leq m}$ e $\left\{V_{i}^{\prime}\right\}_{1 \leq i \leq m}$ satisfazem todas as condições do enunciado.

Na demonstração do Lema 4.9, partição final é construída por meio de sucessivas aplicações do Lema de Regularidade usual, de modo que o número $M_{4.7}$ de partes tem uma forte dependência com o parâmetro $\epsilon$. Em particular, essa demonstração garante apenas que $M_{4.7}$ é limitado por $\operatorname{WOW}\left(\mathcal{E}(0)^{-1}\right)$.

\subsection{Demonstração do Lema da Remoção para Torneios}

Demonstração do Teorema 4.2. Sejam $T$ e $T_{h}$ torneios como no enunciado do teorema. Defina $\mu=\left\lceil 3 \epsilon^{-1}\right\rceil$ e $\mathcal{E}(m)=\min \left\{\gamma, \frac{1}{6} \epsilon\right\}$, onde $\gamma=\gamma_{4.6}\left(\frac{1}{6} \epsilon, h\right)$. Afirmamos que podemos tomar $\delta=$ $\delta_{4.6}\left(\frac{1}{6} \epsilon, \lambda_{4.7}(\mathcal{E}, \mu), h, M_{4.7}(\mathcal{E}, \mu)\right)$.

Aplique o Teorema 4.7 para obter uma equipartição $\mathcal{V}=\left\{V_{1}, \ldots, V_{m}\right\}$ de $T$, com $\mu \leq m \leq$ $M_{4.9}(\mathcal{E}, \mu)$, e uma coleção $\mathcal{V}^{\prime}=\left\{V_{1}^{\prime}, \ldots, V_{m}^{\prime}\right\}$ de conjuntos $V_{i}^{\prime} \subset V_{i} \in \mathcal{V}$, dois a dois $\mathcal{E}(m)$-regulares e com densidades como no enunciado do Teorema.

Considere o torneio $T^{\prime}$ obtido a partir de $T$ após as seguintes alterações:

- Para todos os pares $1 \leq i<j \leq m$ tais que $\vec{d}\left(V_{i}^{\prime}, V_{j}^{\prime}\right)=\vec{d}\left(V_{i}, V_{j}\right) \pm \gamma$, faça $V_{i}$ dominar $^{3} V_{j}$ se $\vec{d}\left(V_{i}, V_{j}\right)<\frac{1}{6} \epsilon$ e faça $V_{j}$ dominar $V_{i}$ se $\vec{d}\left(V_{j}, V_{i}\right)<\frac{1}{6} \epsilon$. Observe que alteramos a orientação de no máximo $m^{2}\left(\frac{1}{6} \epsilon+\frac{1}{6} \epsilon\right) n^{2} / m^{2}=\frac{1}{3} \epsilon n^{2}$ arcos neste caso.

- Para todos os demais pares $i, j$ faça $V_{i}$ dominar $V_{j}$ se $\vec{d}\left(U_{i}, U_{j}\right)<\frac{1}{2}$ ou $V_{j}$ dominar $V_{i}$ caso contrário. Observe que alteramos a orientação de menos que $\mathcal{E}(0) m^{2}\left(n^{2} / m^{2}\right)<\frac{1}{6} \epsilon n^{2} \operatorname{arcos}$ neste processo.

- Para todo $1 \leq i \leq m$, reoriente arcos de forma que $T\left[V_{i}\right]$ se torne um torneio transitivo arbitrário. Observe que menos que $m\left(n^{2} / m^{2}\right) \leq \frac{1}{3} \epsilon n^{2}$ orientações de arcos são alteradas neste processo.

Como $T^{\prime}$ é obtido a partir da reorientação de menos que $\epsilon n^{2} \operatorname{arcos}$ de $T, T^{\prime}$ deve conter ao menos uma cópia de $T_{h}$. Afirmamos que a função

$$
\begin{aligned}
\phi: V\left(T_{h}\right) & \rightarrow \mathcal{V}^{\prime} \\
v & \rightarrow V_{i}^{\prime},
\end{aligned}
$$

\footnotetext{
${ }^{3}$ isto é, reoriente os arcos de forma que todos os vértices de $V_{i}$ dominem todos os vértices de $V_{j}$
} 
onde $i$ é o índice que satisfaz $v \in V_{i}$, atesta que $T_{h} \stackrel{h o m}{\longrightarrow} R:=R_{\epsilon / 6}^{\gamma}\left(T, \mathcal{V}^{\prime}\right)$. De fato, vértices mapeados a um mesmo $V_{i}^{\prime}$ devem formar um torneio transitivo, pois $T^{\prime}\left[V_{i}\right]$ é transitivo. Ademais, vértices $u, v \in V\left(T_{h}\right)$, com $u \rightarrow v$, devem ser tais que $\vec{d}(\phi(u), \phi(v)) \geq \frac{1}{6} \epsilon$, uma vez que não há pares com densidade menor que $\frac{1}{6} \epsilon$ em $T^{\prime}$.

Assim, segue do Lema 4.6 que $T_{h}$ é $\delta$-abundante em $T$.

\subsection{Subtorneios facilmente testáveis}

O Teorema 4.2 afirma que a propriedade Livre $\left(T_{h}\right)$ é testável para todo torneio $T_{h}$ fixo, mas o testador fornecido na demonstração do teorema faz um número de consultas que é WOW $\left(\epsilon^{-1}\right)$. Uma questão natural é se podemos fazer melhor, mesmo que apenas para certos torneios específicos. Mais especificamente, podemos nos perguntar para quais torneios $T_{h}$ a propriedade Livre $\left(T_{h}\right)$ é facilmente testável (para esses torneios dizemos que $T_{h}$ é facilmente testável).

Nessa seção, mostraremos que o conjunto de torneios facilmente testáveis é fechado por operações de expansão (Definição 4.4). Em particular, obtemos que torneios $T_{r, s, t}$, definidos a seguir, são facilmente testáveis.

Definição $4.10\left(\boldsymbol{T}_{\boldsymbol{r}, \boldsymbol{s}, \boldsymbol{t}}\right)$. Seja $T$ um triângulo dirigido, formado por vértices $x, y, z$ satisfazendo $x \rightarrow y \rightarrow z \rightarrow x$. Definimos

$$
T_{r, s, t}=\mathcal{B}_{r}\left(\mathcal{B}_{s}\left(\mathcal{B}_{t}(T, z), y, x\right)\right.
$$

como o torneio obtido a partir da expansão de $x, y, z$ para torneios transitivos de tamanho $r, s$ e $t$, respectivamente. Equivalentemente, $T_{r, s, t}$ é o torneio isomorfo a um torneio transitivo $\pi\left(x_{1}, \ldots x_{r+s+t}\right)$, em que reorientamos arcos de modo que $\left\{x_{r+s+1}, \ldots, x_{r+s+t}\right\}$ domine $\left\{x_{1}, \ldots, x_{r}\right\} . \diamond$

Teorema 4.11. Se $T_{h}$ é um torneio facilmente testável, então $\mathcal{B}_{r}\left(T_{h}, v\right)$ é facilmente testável para qualquer $v \in V\left(T_{h}\right)$ e $r>0$.

Demonstração. Fixe $\epsilon>0$ e seja $T$ um torneio $\epsilon$-longe de $\mathcal{B}_{r}\left(T_{h}, v\right)$. Em particular $T$ deve estar $\epsilon$-longe de $T_{h}$ (pois $T_{h}$ é subgrafo de $T$ ).

Seja $\mathcal{A}$ um $\epsilon$-testador para Livre $\left(T_{h}\right)$ com erro-unilateral. Pelo Teorema 2.23, podemos supor que $\mathcal{A}$ tem acesso apenas ao torneio $T[Q]$ induzido por um conjunto $Q,|Q|=q$, escolhido de maneira uniforme. Como $T_{h}$ é facilmente testável, também podemos supor que $q=\operatorname{poli}\left(\epsilon^{-1}\right)$.

Observamos agora que se $T[Q]$ é livre de $T_{h}$, então $\mathcal{A}$ é obrigado a aceitar $T$. Se esse não fosse o caso, $\mathcal{A}$ teria probabilidade positiva de rejeitar torneios em Livre $\left(T_{h}\right)$. Portanto, com probabilidade pelo menos $\frac{2}{3}, T[Q]$ deve possuir uma cópia de $T_{h}$.

Assim, $T_{h}$ deve ser $\delta$-abundante em $T$, com $\delta \geq \frac{2}{3} q^{-h}=$ poli $_{<}(\epsilon)$. Aplicando o Lema 4.5, concluímos que $\mathcal{B}_{r}\left(T_{h}, v\right)$ é $\delta_{\mathcal{B}}$-abundante em $T$, com $\delta_{\mathcal{B}}=\operatorname{poli}_{<}(\epsilon)$. Daí segue que $\mathcal{B}_{r}\left(T_{h}, v\right)$ é facilmente testável (ver demonstração do Teorema 4.1).

Como o triângulo dirigido é facilmente testável (Teorema 2.26), vale o seguinte resultado.

Colorário 4.12. Para quaisquer $r, s, t>0$, o torneio $T_{r, s, t}$ é facilmente testável. 


\section{Capítulo 5}

\section{Caracterização das propriedades testáveis com erro-unilateral}

Mostraremos neste capítulo que uma propriedade $\mathcal{P}$ de torneios é testável com erro-unilateral se, e somente se, $\mathcal{P}$ é semi-hereditária. Propriedades semi-hereditárias serão definidas, posteriormente, como uma relaxação de propriedades hereditárias, definidas a seguir.

Definição 5.1 (propriedade hereditária). Uma propriedade de torneios $\mathcal{H}$ é hereditária se $\mathcal{H}$ é fechada por subtorneios, isto é, se $T \in \mathcal{H}$ implica $T^{\prime} \in \mathcal{H}$ para todo $T^{\prime}$ subtorneio de $T$.

\subsection{Testando famílias infinitas de subtorneios}

Definição $5.2(\operatorname{Livre}(\mathcal{F}))$. Seja $\mathcal{F}$ uma família (possivelmente infinita) de torneios. Dizemos que um torneio $T$ é livre de $\mathcal{F}$ se, para todo $F \in \mathcal{F}, T$ é livre de $F$. Definimos a propriedade Livre $(\mathcal{F})$ formada por todos os torneios livres de $\mathcal{F}$.

Notamos que para toda propriedade hereditária $\mathcal{H}$, existe uma família $\mathcal{F}$ tal que $\mathcal{H}=\operatorname{Livre}(\mathcal{F})$. De fato, como permitimos $\mathcal{F}$ ser infinita, basta tomar $\mathcal{F}$ como a família de todos os torneios que não satisfazem $\mathcal{H}$. O fato de toda propriedade hereditária ser testável com erro-unilateral segue, portanto, como corolário do seguinte resultado.

Teorema 5.3 (Lema da Remoção para Famílias de Torneios [9] $]_{g r}$ ). Para qualquer família $\mathcal{F}$ de torneios e qualquer $0<\epsilon<1$, existe um $\delta=\delta_{5.3}(\epsilon, \mathcal{F})>0$ e um inteiro $S=S_{5.3}(\epsilon, \mathcal{F})$ tal que: se $T$ é um torneio $\epsilon$-longe de Livre $(\mathcal{F})$, então existe algum $F \in \mathcal{F}$, de tamanho no máximo $S$, que é $\delta$-abundante em $T$.

Para famílias $\mathcal{F}$ finitas o resultado acima é trivial, bastando aplicar o Teorema 4.2 para todo $F \in \mathcal{F}$ e tomar $\delta=\min _{F \in \mathcal{F}} \delta_{4.2}(\epsilon, F)$.

Para famílias de cardinalidade infinita, podemos repetir o mesmo raciocínio feito na demonstração do Teorema 4.2 para concluir que existe um $F \in \mathcal{F}$ tal que $F \stackrel{\text { hom }}{\longrightarrow} R$, onde $R$ é o digrafo reduzido associado a uma coleção $\mathcal{V}^{\prime}$ de conjuntos de vértices dois a dois regulares de $T$. Mas como não sabemos, a priori, o tamanho deste torneio $F$, não há como determinar um valor absoluto para o quão regular $\mathcal{V}^{\prime}$ deve ser.

Por outro lado, o Lema Forte da Regularidade nos permite condicionar o quão regular o objeto devolvido deve ser em função de seu tamanho $m$. A seguinte proposição nos auxilia nesse sentido. 
Proposição 5.4. Para toda família $\mathcal{F}$ de torneios e inteiro $m$, existe uma função $\psi_{\mathcal{F}}(m): \mathbb{N} \rightarrow \mathbb{N}$

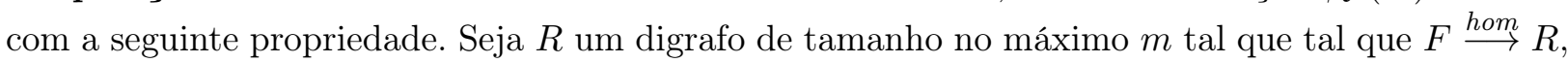
para algum $F \in \mathcal{F}$. Então existe $F^{\prime} \in \mathcal{F}$, de tamanho no máximo $\psi_{\mathcal{F}}(m)$, tal que $F^{\prime} \stackrel{\text { hom }}{\longrightarrow} R$.

Demonstração. Considere a seguinte família de digrafos:

$$
\mathcal{F}_{m}=\{R:|R| \leq m \text { e existe } F \in \mathcal{F} \text { tal que } F \stackrel{\text { hom }}{\longrightarrow} R\}
$$

Como a família $\mathcal{F}_{m}$ é finita e $R \in \mathcal{F}_{m}$ então podemos simplesmente tomar $\psi_{\mathcal{F}}(m)$ como

$$
\psi_{\mathcal{F}}(m)=\max _{R \in \mathcal{F}_{m}} \min _{F \in \mathcal{F}: F \stackrel{\text { hom }}{\longrightarrow} R}|F|
$$

Demonstração do Teorema 5.3. Defina $\mu=\left\lceil 3 \epsilon^{-1}\right\rceil$ e

$$
\mathcal{E}(m)=\min \left\{\gamma_{4.6}\left(\frac{1}{6} \epsilon, \psi_{\mathcal{F}}(m)\right), \frac{1}{6} \epsilon\right\}
$$

Mostraremos que podemos tomar

$$
\delta=\delta_{4.6}\left(\frac{1}{6} \epsilon, \lambda_{4.7}(\mathcal{E}, \mu), \psi_{\mathcal{F}}(m), M_{4.7}(\mathcal{E}, \mu)\right)
$$

Notamos que as definições acima são idênticas às da prova do Teorema 4.2, com a diferença de que o tamanho $h$ dos torneios lá considerados foi substituído por $\psi_{\mathcal{F}}(m)$. Seguimos esta demonstração de forma similar.

Aplique o Teorema 4.7 para obter uma equipartição $\mathcal{V}=\left\{V_{1}, \ldots, V_{m}\right\}$ de $T$ de cardinalidade $\mu \leq m \leq M_{4.7}(\mathcal{E}, \mu)$ e uma coleção $\mathcal{V}^{\prime}=\left\{V_{1}^{\prime}, \ldots, V_{m}^{\prime}\right\}$ de conjuntos $V_{i}^{\prime} \subset V_{i}$, dois a dois $\mathcal{E}(m)$ regulares e com densidades como no enunciado do teorema.

Considere um torneio $T^{\prime}$, obtido a partir de $T$ por meio das seguintes alterações.

- Para todos os pares $1 \leq i<j \leq m$ tais que $\vec{d}\left(V_{i}^{\prime}, V_{j}^{\prime}\right)=\vec{d}\left(V_{i}, V_{j}\right) \pm \gamma$, faça $V_{i}$ dominar $^{1} V_{j}$ se $\vec{d}\left(V_{i}, V_{j}\right)<\frac{1}{6} \epsilon$ e faça $V_{j}$ dominar $V_{i}$ se $\vec{d}\left(V_{j}, V_{i}\right)<\frac{1}{6} \epsilon$. Observe que alteramos a orientação de no máximo $m^{2}\left(\frac{1}{6} \epsilon+\frac{1}{6} \epsilon\right) n^{2} / m^{2}=\frac{1}{3} \epsilon n^{2}$ arcos neste caso.

- Para todos os demais pares $i, j$ faça $V_{i}$ dominar $V_{j}$ se $\vec{d}\left(V_{i}^{\prime}, V_{j}^{\prime}\right)<\frac{1}{2}$ ou $V_{j}$ dominar $V_{i}$ caso contrário. Observe que alteramos a orientação de menos que $\mathcal{E}(0) m^{2}\left(n^{2} / m^{2}\right)<\frac{1}{6} \epsilon n^{2} \operatorname{arcos}$ neste processo.

- Para todo $1 \leq i \leq m$, reoriente arcos de forma que $T\left[V_{i}\right]$ se torne um torneio transitivo arbitrário. Observe que menos que $m\left(n^{2} / m^{2}\right) \leq \frac{1}{3} \epsilon n^{2}$ orientações de arcos são alteradas neste processo.

Como $T^{\prime}$ é obtido a partir da reorientação de menos que $\epsilon n^{2} \operatorname{arcos}$ de $T$, segue que $T^{\prime}$ deve conter ao menos uma cópia de algum $F \in \mathcal{F}$. Pela escolha dos arcos reorientados, a função

$$
\begin{aligned}
\phi: V(F) & \rightarrow \mathcal{V}^{\prime} \\
v & \mapsto V_{i}^{\prime},
\end{aligned}
$$

\footnotetext{
${ }^{1}$ isto é, reoriente os arcos de forma que todos os vértices de $V_{i}$ dominem todos os vértices de $V_{j}$.
} 
onde o índice $i$ satisfaz $v \in V_{i}$, atesta que $F \stackrel{h o m}{\longrightarrow} R:=R_{\epsilon / 6}^{\gamma}\left(T, \mathcal{V}^{\prime}\right)$. Como $|R|=m$, a Proposição 5.4 nos garante a existência de um $F^{\prime} \in \mathcal{F},\left|F^{\prime}\right| \leq \phi_{\mathcal{F}}(m)$, tal que $F^{\prime} \stackrel{\text { hom }}{\longrightarrow} R$. A restrição sobre o tamanho de $F^{\prime}$ nos permite aplicar o Lema 4.6 para concluir que $F^{\prime}$ é $\delta$-abundante em $T$.

Como discutido no início da seção, o Teorema 5.3 implica o seguinte o resultado.

Teorema $5.5\left([9]_{g r}\right)$. Toda propriedade hereditária de torneios é testável com erro-unilateral.

Observamos que o resultado acima não garante que para qualquer propriedade hereditária $\mathcal{H}$ exista um testador para $\mathcal{H}$ cujo parâmetro $\epsilon$ faz parte da entrada, uma vez que não há garantias que a função $\phi_{\mathcal{F}}$ associada seja sequer computável. Na verdade, é possível mostrar que de fato existem (ver [9] para grafos ) propriedades hereditárias (decidíveis) para as quais não é possível computar o número de consultas necessárias para testá-la como função de $\epsilon$.

Como veremos adiante, uma versão aproximada da recíproca do Teorema 5.5 é verdadeira. Isto é, as propriedades testáveis com erro-unilateral são exatamente as hereditárias, a menos de uma relaxação natural dentro do contexto de teste de propriedades.

\subsection{Propriedades semi-hereditárias}

Definição 5.6 (propriedade semi-hereditária). Uma propriedade de torneios $\mathcal{P}$ é dita semihereditária se existe uma propriedade hereditária $\mathcal{H}$ satisfazendo as seguinte condições:

(i) Todo torneio que satisfaz $\mathcal{P}$ também satisfaz $\mathcal{H}(\mathcal{P} \subseteq \mathcal{H})$.

(ii) Existe uma função $M:(0,1) \rightarrow \mathbb{N}$ tal que para todo $\epsilon>0$, qualquer torneio $\epsilon$-longe de $\mathcal{P}$ e de tamanho maior que $M(\epsilon)$ não satisfaz $\mathcal{H}$ (e portanto contém uma cópia induzida de algum membro de $\mathcal{F}_{\mathcal{H}}$ ).

Em outras palavras, uma propriedade semi-hereditária $\mathcal{P}$ é um subconjunto de alguma propriedade hereditária $\mathcal{H}$, com a restrição de que para todo $\epsilon$ existe apenas um número finito de torneios $\epsilon$-longe de $\mathcal{P}$ que satisfazem $\mathcal{H}$.

Note como a segunda condição da definição acima implica, em particular, que um torneio (suficientemente grande) $T_{n} \epsilon$-longe de $\mathcal{P}$ também está, digamos, $\frac{\epsilon}{2}$-longe de $\mathcal{H}$. De fato, qualquer torneio obtido pela reorientação de $\frac{\epsilon}{2} n^{2}$ arestas de $T_{n}$ ainda está $\frac{\epsilon}{2}$-longe de $\mathcal{P}$ e, portanto, não pertence a $\mathcal{H}$ se $n>M\left(\frac{\epsilon}{2}\right)$.

Neste sentido, o conceito de propriedades semi-hereditárias é uma relaxação natural de hereditariedade no contexto de teste de propriedades. O resultado a seguir mostra que as propriedades testáveis com erro-unilateral são exatamente as semi-hereditárias.

Teorema $5.7\left([9]_{\mathrm{gr}}\right)$. Uma propriedade $\mathcal{P}$ é testável ${ }^{2}$ com erro-unilateral se, e somente se, $\mathcal{P}$ é semi-hereditária.

\footnotetext{
${ }^{2}$ ver Observação 2.24 .
} 
Demonstração. Primeiro, podemos construir um $\epsilon$-testador para uma propriedade semi-hereditária $\mathcal{P}$ simplesmente usando - para torneios suficientemente grandes - um $\frac{1}{2} \epsilon$-testador para a propriedade hereditária $\mathcal{H}$ associada. De fato, torneios que satisfazem $\mathcal{P}$ também satisfazem $\mathcal{H}$ e, pela observação feita anteriormente, torneios $\epsilon$-longe de $\mathcal{P}$ também estão $\frac{1}{2} \epsilon$-longe de $\mathcal{H}$.

Reciprocamente, seja $\mathcal{P}$ uma propriedade testável com erro-unilateral. Para todo $\epsilon$, fixe um $\epsilon$-testador não-adaptativo $\mathcal{A}_{\epsilon}$ para $\mathcal{P}$ e defina $q_{\epsilon}$ como o número de vértices que $\mathcal{A}_{\epsilon}$ amostra.

Seja $\mathcal{F}$ a família composta pelos torneios $F$ para os quais existe um $\epsilon$ tal que

- o tamanho de $F$ é exatamente $q_{\epsilon}$ e

- o algoritmo $\mathcal{A}_{\epsilon}$ rejeita sua entrada quando o torneio amostrado é isomorfo a $F$.

Afirmamos que $\mathcal{P}$ é uma propriedade semi-hereditária associada à propriedade $\mathcal{H}:=\operatorname{Livre}(\mathcal{F})$, $\operatorname{com} M(\epsilon)=q_{\epsilon}$.

Primeiro precisamos mostrar que $\mathcal{P} \subseteq \mathcal{H}$. Suponha que exista um torneio $T \in \mathcal{P}$ tal que $T \notin \mathcal{H}$. Então $T$ contém uma cópia de algum $F \in \mathcal{F}$. Pela definição de $\mathcal{F}$, existe $\epsilon$ tal que o número $q_{\epsilon}$ de vértices amostrados por $\mathcal{A}_{\epsilon}$ é exatamente $|F|$. Portanto, com probabilidade positiva, o subtorneio amostrado por $\mathcal{A}_{\epsilon}$ é isomorfo a $F$. Logo $\mathcal{A}_{\epsilon}$ não é um testador com erro-unilateral, uma vez que $T$ é rejeitado com probabilidade positiva.

Resta provar que $\mathcal{P}$ atende à segunda condição da Definição 5.6. Seja $T$ um torneio $\epsilon$-longe de $\mathcal{P}$ e de tamanho pelo menos $M(\epsilon)=q_{\epsilon}$. Considere uma execução do algoritmo $\mathcal{A}_{\epsilon}$ em que $T$ é rejeitado (lembrando que $T$ é rejeitado com probabilidade $\frac{2}{3}$ ). O torneio $F$ amostrado nessa execução está contido em $\mathcal{F}$, por definição. Mas como $F$ é um subtorneio de $T$, concluímos que $T \notin \operatorname{Livre}(\mathcal{F})=\mathcal{H}$. 


\section{Capítulo 6}

\section{Caracterização de propriedades testáveis}

Após termos caracterizado as propriedades de torneio testáveis com erro-unilateral (Teorema 5.7), é natural nos perguntarmos quais são exatamente as propriedades testáveis, em condições genéricas. Em um primeiro momento, não é sequer evidente que existam propriedades não testáveis. Veremos a seguir que tais propriedades, de fato, existem. Em particular, veremos que não é possível testar isomorfismo entre torneios.

\subsection{Exemplo de propriedade não testável}

Definição 6.1 (propriedade Iso). Considere a seguinte propriedade Iso de torneios. Um torneio $T=(V, A)$ satisfaz a propriedade Iso se

(i) $V$ pode ser particionado em dois conjuntos $U_{+}, U_{-}$tais que todo vértice de $U_{+}$domina todos os vértices de $U_{-}$e

(ii) $T\left[U_{+}\right]$e $T\left[U_{-}\right]$são isomorfos.

Teorema 6.2 ([17]). A propriedade Iso não é testável.

Para provar o Teorema 6.2, mostraremos que existe um par de torneios que estão longe de serem isomorfos, mas que possuem exatamente o mesmo número de cópias de subtorneios de tamanho $q$, para qualquer $q$ fixado a priori. Isso implica que um testador que faz amostras de tamanho $q$ "enxerga" esses dois torneios exatamente da mesma forma, isto é, ambos têm a mesma probabilidade de serem aceitos ou rejeitados.

Lema 6.3 ([17]). Existe uma constante $\epsilon=\epsilon_{6.3}>0$ tal que para todo inteiro $q>0$, existem torneios $T_{r}$ e $T_{r}^{\prime}$, com $r=r_{6.3}(q)$, satisfazendo as seguintes propriedades:

(i) $T_{r}^{\prime}$ está $\epsilon$-longe de ser isomorfo a $T_{r}$.

(ii) $T_{r}$ e $T_{r}^{\prime}$ têm o mesmo número de cópias de $H$, para qualquer torneio $H$ de tamanho menor ou igual a $q$. 
(iii) Ambos os torneios $T_{r}$ e $T_{r}^{\prime}$ são tais que todo vértice tem grau de saída e entrada pelo menos $\frac{1}{6} r$ e, para qualquer subconjunto de vértices $S$ tal que $\frac{1}{6} r \leq|S| \leq \frac{1}{2} r$, vale que

$$
\vec{e}(S, V \backslash S) \geq \frac{1}{72} r^{2} \quad \quad \quad \vec{e}(V \backslash S, S) \geq \frac{1}{72} r^{2} .
$$

Demonstração. Seja $U=22^{\left(\begin{array}{c}r \\ 2\end{array}\right)}$ o número de torneios com $r$ elementos.

Primeiramente, vamos mostrar que pelo menos $\frac{1}{2} U$ torneios de tamanho $r$ satisfazem a terceira condição acima. De fato, escolha um torneio $T$ de tamanho $r$ uniformemente ao acaso e fixe um vértice $v$ de $T$. Temos

$$
\mathbb{P}\left(g^{+}(v)<\frac{1}{2} r-\frac{1}{3} r\right) \underset{\text { A. } 2}{\leq} e^{\frac{-2(r / 3)^{2}}{r}}=e^{\frac{-2 r}{9}} .
$$

Além disso, fixado $S \subset V(T), \frac{1}{6} r \leq|S| \leq \frac{1}{2} r$, temos

$$
\mathbb{P}\left(\vec{e}(S, V \backslash S) \leq \frac{5}{72} r^{2}-\frac{1}{18} r^{2}\right) \underset{\text { A.2 }}{\leq} e^{\frac{-2\left(r^{2} / 18\right)^{2}}{5 r^{2} / 36}}=e^{-\frac{2}{45} r^{2}} .
$$

Assim, a probabilidade de $T$ não satisfazer a terceira propriedade é no máximo

$$
2\left(r e^{-\frac{2}{9} r}+2^{\frac{1}{2} r} e^{-\frac{2}{45} r^{2}}\right)<\frac{1}{2},
$$

para $r$ suficientemente grande.

Existem menos que $q 2^{q^{2}}$ torneios $H$ de tamanho no máximo $q$. Para cada uma desses torneios $H, T_{r}$ pode conter no máximo $r^{q}$ cópias de $H$. Colocando $C=q^{2} 2^{q^{2}}$, concluímos que há no máximo ${ }_{r}^{C}$ configurações possíveis para a contagem das cópias de torneios $H$ em torneios de tamanho $r$. Pelo princípio da casa dos pombos, devem existir pelo menos $\frac{1}{2} \frac{U}{r^{C}}$ torneios satisfazendo as duas últimas propriedades do lema.

Finalmente, fixe um tal torneio $T_{r}$ e uma constante $\epsilon>0$. O número total de torneios que estão $\epsilon$-perto de serem isomorfos a $T_{r}$ é no máximo

$$
\left.\left.r ! \sum_{i=0}^{\epsilon r^{2}}\left(\begin{array}{c}
r \\
2
\end{array}\right)\right)<r !\left(\begin{array}{c}
r^{2} \\
\epsilon
\end{array}\right)<r^{2}\right)<r^{r}\left(e \epsilon^{-1}\right)^{\epsilon r^{2}}
$$

Escolhendo $\epsilon$ tal que $\left(e \epsilon^{-1}\right)^{\epsilon}<2^{-\frac{1}{4}}$, temos que o número de tais torneios é menor que $2^{r \log r+r^{2} / 4}$, que é menor que $\frac{1}{2} \frac{U}{r^{C}}$, para $r$ suficientemente grande. Concluímos então que existe um torneio $T_{r}^{\prime}$ que, junto com $T_{r}$, satisfaz todas as condições do lema.

Demonstração do Teorema 6.2. Suponha por absurdo que Iso seja testável e considere um $\epsilon$-testador para Iso, com $\epsilon=\frac{1}{36} \epsilon_{6.3}^{2}$. O Teorema 2.23 nos permite assumir que esse testador faz sua decisão a partir do torneio induzido por um conjunto de $q$ vértices escolhidos uniformemente ao acaso dentre os vértices do torneio de entrada, para um certo $q \geq 0$.

Seja $r=r_{6.3}(q)$ e tome torneios $T_{r}$ e $T_{r}^{\prime}$ como no enunciado do Lema 6.3. Construa um torneio $T_{\text {sim }}$ composto por uma cópia de $T_{r}$ cujos vértices dominam todos os vértices de uma segunda cópia (disjunta) de $T_{r}$. Construa, ainda, um torneio $T_{n a o}$ composto por uma cópia de $T_{r}$ cujos vértices dominam todos os vértices de uma cópia (disjunta) de $T_{r}^{\prime}$.

É evidente que $T_{\text {sim }}$ satisfaz a propriedade Iso. Afirmamos que $T_{n a o}$ está $\epsilon$-longe de Iso. De fato, suponha que exista um torneio $\epsilon$-próximo a $T_{n a o}$, admitindo conjuntos $U_{+}$e $U_{-}$como na 
propriedade Iso. Seja $k=\left|U_{+} \cap V\left(T_{r}^{\prime}\right)\right|$. Como é necessário inverter $k^{2}$ arcos de $T_{n a o}$ para que $U_{+}$ domine $U_{-}$, devemos ter $k \leq \frac{1}{3} \epsilon_{6.3} r$. Mas a existência de um torneio $\epsilon$-próximo a $T_{\text {nao }}$ que satisfaz Iso implica ser possível tornar $T_{r}$ e $T_{r^{\prime}}$ isomorfos após a inversão de menos que

$$
\epsilon(2 r)^{2}+k^{2}+k r<\left(4 \epsilon+\frac{2}{3} \epsilon_{6.3}\right) r^{2}<\epsilon_{6.3} r^{2}
$$

arcos, um absurdo.

Assim, todo $\epsilon$-testador deve aceitar $T_{s i m}$ e rejeitar $T_{\text {nao }}$, com probabilidade pelo menos $\frac{2}{3}$, respectivamente. Por outro lado, como $T_{\text {sim }}$ e $T_{\text {nao }}$ têm o mesmo número de cópias de cada torneio de tamanho $q$, a probabilidade do testador considerado no começo da demonstração aceitar $T_{\text {sim }}$ deve ser exatamente a mesma de aceitar $T_{n a o}$. Segue, portanto, que esse tal $\epsilon$-testador não pode existir. 


\section{Capítulo 7}

\section{Considerações finais}

Neste trabalho, verificamos que alguns dos resultados conhecidos a respeito de teste de propriedades em grafos e digrafos possuem versões análogas para o caso de torneios.

Alon, Fischer, Krivelevich e Szegedy mostraram em [3] que é possível testar se grafos são livres de cópias de um certo subgrafo $H$ com erro-unilateral, não apenas quando consideramos cópias quaisquer mas também quando nos restringimos apenas a cópias induzidas. Demonstrar tal afirmação constituiu um passo crucial na direção de caracterizar as propriedades testáveis em grafos, não apenas pelo resultado em si, mas principalmente pelas ferramentas empregadas para atingi-lo, como o Lema Forte da Regularidade. Nos capítulos 3 e 4, vimos que técnicas análogas podem ser usadas para provar que a propriedade de torneios Livre $\left(T_{h}\right)$ - formada pelos torneios livres de cópias de um torneio $T_{h}$ fixo - é facilmente testável com erro-unilateral. Em particular, provamos um Lema de Remoção para Torneios (4.2) que afirma que torneios que estão longe de serem livres de $T_{h}$ possuem uma densidade positiva de cópias de $T_{h}$, de forma que tais "testemunhas" podem ser encontradas com alta probabilidade em uma amostra de tamanho constante da entrada. Salientamos que a dificuldade de provar tal afirmação é similar à dificuldade da versão para grafos que considera apenas cópias induzidas, no sentido que não parece existir uma forma imediata de fazê-lo usando apenas a versão mais simples do Lema de Regularidade (Teorema 3.25).

Mostramos ainda que, assim como ocorre no caso de grafos e digrafos [9], todas as propriedades hereditárias de torneios são testáveis e que, a menos de uma generalização natural, essas são as únicas testáveis com erro-unilateral. No capítulo 6, apresentamos uma propriedade de torneios que não pode ser testada (com ou sem erro-unilateral). A propriedade apresentada explora a dificuldade de testar isomorfismo entre torneios. Tal dificuldade é uma consequência da existência de torneios que, apesar de estarem longe de ser isomorfos, têm exatamente a mesma quantidade de cada subtorneio de tamanho fixo $q$ (o que implica que um testador que faz amostras de tamanho $q$ os enxerga exatamente da mesma forma). Essa dificuldade também existe para o caso de grafos [3].

Por outro lado, encontramos ao menos um problema em testes de propriedades de torneios que, aparentemente, não pode ser resolvido como nos casos análogos para grafos e digrafos, a saber, o problema de determinar quais são os torneios facilmente testáveis. Na seção a seguir, discutimos esse problema de forma mais elaborada. Por fim, enunciamos na seção subsequente dois teoremas que acreditamos serem verdadeiros para torneios, mas que não foram desenvolvidos neste trabalho. 


\subsection{Subtorneios facilmente testáveis}

No estudo de teste de propriedades em grafos, fixado um grafo $H$, podemos considerar a propriedade de um grafo "ser livre de cópias de $H$ ", análoga à propriedade $\operatorname{Livre}\left(T_{h}\right)$ para torneios. Se essa propriedade é facilmente testável, dizemos que $H$ é facilmente testável. Diferentemente do caso de torneios, existem duas versões dessa propriedade: a primeira é composta por grafos que não possuem $H$ como subgrafo; a segunda é composta por grafos que não possuem $H$ como subgrafo induzido.

Ao tratarmos de cópias não necessariamente induzidas, sabe-se que um grafo $H$ é facilmente testável se, e somente se, $H$ for bipartido [2]. No caso de cópias induzidas, o problema também foi resolvido (quase que) completamente por Alon e Shapira [6, 8]:

- os caminhos $P_{2}, P_{3}, P_{4}$ (de comprimento $1,2,3$ ) e seus respectivos complementos $\overline{P_{2}}, \overline{P_{3}}, \overline{P_{4}}$ são facilmente testáveis;

- não se sabe se o circuito $C_{4}$ (de comprimento 4 ) ou se $\overline{C_{4}}$ são testáveis;

- todos os demais grafos não são facilmente testáveis.

Existem resultados similares para o caso de digrafos [7, 8]. Em particular, Alon e Shapira [8] mostraram que triângulos (dirigidos) não são facilmente testáveis em digrafos, o que contrasta com o resultado oposto apresentado para torneios no Teorema 2.26. A demonstração desses autores utiliza uma construção de Behrend sobre conjuntos densos sem progressões aritméticas de tamanho 3 para provar a existência de digrafos que, apesar de serem $\epsilon$-longe de serem livres de triângulos, não são poli $(\epsilon)$-abundantes de triângulos. Todos as demonstrações (contidas nos artigos supracitados) que provam que certos grafos ou digrafos não são facilmente testáveis também se baseiam em construções bem similares. Os grafos (ou digrafos) resultantes de tais construções têm densidade de arestas (ou arcos) bem menor que 1 . Nesse sentido, mostrar que certo torneio $T_{h}$ não é facilmente testável parece ser uma tarefa mais complexa.

Na outra direção, mostramos que o conjunto $\mathcal{F} \mathcal{T}$ formado pelos torneios facilmente testáveis é fechado por operações de expansão (Teorema 4.11), o que implica que se $T^{\prime}$ é facilmente testável e $T \stackrel{\text { hom }}{\longrightarrow} T^{\prime}$, então $T$ é facilmente testável. Em particular, garantimos a existência de um número infinito de torneios facilmente testáveis, o que contrasta com os caso de cópias induzidas em grafos e digrafos. Neste contexto, uma questão que nos parece interessante é se o conjunto de torneios facilmente testáveis é finito "a menos de operações de homomorfismo".

Pergunta 7.1. Existe um conjunto finito $S$, tal que $\mathcal{F} \mathcal{T}=\left\{T: T \stackrel{h o m}{\longrightarrow} T^{\prime}\right.$, para algum $\left.T^{\prime} \in S\right\}$ ? $\diamond$

Por fim, notamos que grafos bipartidos são facilmente testáveis ${ }^{1}$ simplesmente porque todo grafo bipartido fixo é poli $(\epsilon)$-abundante em grafos com densidade de arestas pelo menos $\epsilon{ }^{2}$ De maneira similar, o fato de torneios $T_{r, s, t}$ serem facilmente testáveis é uma consequência direta de $T_{r, s, t}$ ser poli $\left(\epsilon^{\prime}\right)$-abundante em qualquer torneio com densidade pelo menos $\epsilon^{\prime}$ de triângulos orientados - note que torneios $\epsilon$-longe de $\operatorname{Livre}\left(T_{h}\right)$ têm densidade pelo menos $\epsilon^{\prime}:=\operatorname{poli}(\epsilon)$ de triângulos orientados, para todo $T_{h}$ não transitivo ${ }^{3}$. Parece portanto plausível formular a seguinte questão.

Pergunta 7.2. Podemos tomar o conjunto $S$ da Pergunta 7.1 como um conjunto unitário contendo um triângulo orientado?

\footnotetext{
${ }^{1}$ no caso não induzido.

${ }^{2}$ note que ser $\epsilon$-longe de ser livre de qualquer grafo $H$ implica ter densidade de arestas pelo menos $\epsilon$.

${ }^{3}$ não existem torneios (suficientemente grandes) $\epsilon$-longe de $T_{h}$ acíclicos.
} 


\subsection{Resultados adicionais para torneios}

Alon, Fischer, Newman e Shapira [5] deram uma caracterização das propriedades testáveis em grafos (com ou sem erro-unilateral) que mostra que testabilidade e regularidade são conceitos intrinsecamente relacionados.

De maneira informal, os autores mostraram que uma propriedade $\mathcal{P}$ de grafos é testável se, e somente se, existe um conjunto finito $\mathcal{C}$ de "classes" de partições regulares satisfazendo as seguinte condições: i) grafos que satisfazem $\mathcal{P}$ admitem partições regulares pertencentes a alguma classe $C \in \mathcal{C}$; ii) grafos longe de $\mathcal{P}$ estão longe de admitirem partições em $C$, para todo $C \in \mathcal{C}$. A seguir, fazemos as definições necessárias para formalizar tais idéias para o caso de torneios.

Definição 7.3 (classe de partições). Uma classe de partições $C$ é uma 4-upla

$$
\left(\gamma, m,\left\{\eta_{i j}\right\}_{1 \leq i<j \leq m}, I\right)
$$

tal que $0<\gamma \leq 1,0 \leq \eta_{i j} \leq 1, m$ é um inteiro positivo e $I$ é um conjunto de no máximo $\gamma m^{2}$ pares $(i, j)$ com $1 \leq i<j \leq m$. Dizemos que um torneio $T$ satisfaz a classe $C$ se existe uma equipartição $\left\{V_{1}, \ldots, V_{m}\right\}$ dos vértices de $T$ tal que, para todo $(i, j) \notin I$, o par $\left(V_{i}, V_{j}\right)$ é $\epsilon$-regular com densidade $\vec{d}\left(V_{i}, V_{j}\right)=\eta_{i j}(1 \leq i<j \leq m)$.

Definição 7.4 (classe-redutível). Uma propriedade de torneios $\mathcal{P}$ é classe-redutível se para todo $\theta>0$, existe uma coleção finita $\mathcal{C}$ de classes de equipartições tais que para todo $\epsilon>0$ e todo torneio $T$ satisfazem as seguintes condições:

1. Se $T$ satisfaz $\mathcal{P}$, então $T$ está $\theta$-perto de satisfazer $C$, para algum $C \in \mathcal{C}$.

2. Se $T$ está $\epsilon$-longe de $\mathcal{P}$, então para todo $C \in \mathcal{C}$. $T$ está $(\epsilon-\theta)$-longe de satisfazer $C$, para todo $C \in \mathcal{C}$.

Acreditamos ser possível demonstrar o seguinte resultado procedendo de maneira análoga à demonstração contida em [5].

Conjectura 7.5. Uma propriedade de torneios $\mathcal{P}$ é testável se, e somente se, $\mathcal{P}$ é classe-redutível. $\diamond$

Usando técnicas similares às empregadas na demonstração do resultado acima para grafos, Fischer e Newman [18] provaram que é possível estimar a distância de um grafo a qualquer propriedade testável. A seguir, formalizamos os conceitos relacionados a essa afirmação para o caso de torneios.

Definição $7.6((\boldsymbol{\epsilon}, \boldsymbol{\theta})$-estimável). Uma propriedade de torneios $\mathcal{P}$ é $(\epsilon, \theta)$-estimável se existe um algoritmo de decisão probabilístico que recebe acesso a um torneio $T$ de entrada por meio de um oráculo e satisfaz as seguintes propriedades.

1. Se $T$ está $\left(\epsilon-\frac{1}{2} \theta\right)$-perto de $\mathcal{P}$, então $\mathcal{A}$ aceita $T$ com probabilidade pelo menos $\frac{2}{3}$.

2. Se $T$ está $\left(\epsilon+\frac{1}{2} \theta\right)$-longe de $\mathcal{P}$, então $\mathcal{A}$ rejeita $T$ com probabilidade pelo menos $\frac{2}{3}$.

3. O número máximo de consultas que $\mathcal{A}$ faz ao oráculo independe do tamanho de $T$.

Dizemos que uma propriedade é estimável se ela for $(\epsilon, \theta)$-estimável para todo $\epsilon>0$ e $\theta>0$. 
Observamos que dada uma propriedade $\mathcal{P}$ estimável, um torneio $T$ e uma constante $\theta>0$, é possível estimar a distância de $T$ a $\mathcal{P}$ (a menos de um erro $\theta$ ) por meio de uma busca binária que executa algoritmos como acima descritos para diversos valores de $\epsilon$.

Acreditamos que o seguinte resultado pode ser provado de forma similar à feita em [18] para grafos.

Conjectura 7.7. Toda propriedade testável de torneios é estimável. 


\section{Apêndice A}

\section{Desigualdade de Chebyshev e Cotas de Chernoff}

Seja $Z$ uma variável aleatória qualquer com média $\mu$ e variância $\sigma^{2}$ finitas. Não é difícil verificar que vale a seguinte desigualdade.

Teorema A.1 (Desigualdade de Chebyshev).

$$
\mathbb{P}(|Z-\mu| \geq k \sigma) \leq \frac{1}{k^{2}}
$$

Seja $X$ uma variável com distribuição binomial com parâmetros $n$ e $p$ e seja $\mu=\mathbb{E}(X)=n p$. Os dois resultados abaixo mostram que a probabilidade de $X$ desviar de $\mu$ por um fator aditivo $a$ decresce exponencialmente em função de $a$. Estas e outras cotas de desvios de somas de variáveis independentes em relação a média - usualmente denominadas cotas de Chernoff — podem ser obtidas a partir das desigualdades contidas em [13] (consultar também [10, Apêndice A]).

Teorema A.2 (Cota de Chernoff). Para todo $0<a<\mu$,

$$
\begin{array}{r}
\mathbb{P}(X \geq \mu+a) \leq e^{\frac{a^{2}}{3 \mu}} \\
e \mathbb{P}(X \leq \mu-a) \leq e^{\frac{a^{2}}{2 \mu}}
\end{array}
$$

Colorário A.3 (Cota de Chernoff). Para todo $0<a<\mu$,

$$
\mathbb{P}(|X-\mathbb{E}(X)| \geq a) \leq 2 e^{\frac{a^{2}}{3 \mu}} .
$$




\section{Referências Bibliográficas}

[1] N. Alon, R. A. Duke, H. Lefmann, V. Rödl, and R. Yuster. The algorithmic aspects of the regularity lemma. J. Algorithms, 16(1):80-109, January 1994. 26

[2] Noga Alon. Testing subgraphs in large graphs. Random Structures Algorithms, 21(3-4):359370, 2002. Random structures and algorithms (Poznan, 2001). 11, 40

[3] Noga Alon, Eldar Fischer, Michael Krivelevich, and Mario Szegedy. Efficient testing of large graphs. Combinatorica, 20(4):451-476, 2000. 23, 26, 39

[4] Noga Alon, Eldar Fischer, Ilan Newman, and Asaf Shapira. A combinatorial characterization of the testable graph properties: It's all about regularity. In Proceedings of the Thirty-eighth Annual ACM Symposium on Theory of Computing, STOC '06, pages 251-260, New York, NY, USA, 2006. ACM. 12

[5] Noga Alon, Eldar Fischer, Ilan Newman, and Asaf Shapira. A combinatorial characterization of the testable graph properties: It's all about regularity. SIAM J. Comput., 39(1):143-167, May 2009. 2, 41

[6] Noga Alon and Jacob Fox. Testing perfection is hard, 2014. 40

[7] Noga Alon and Asaf Shapira. Testing subgraphs in directed graphs. J. Comput. Syst. Sci., 69(3):354-382, November 2004. 14, 18, 40

[8] Noga Alon and Asaf Shapira. A characterization of easily testable induced subgraphs. Comb. Probab. Comput., 15(6):791-805, November 2006. 40

[9] Noga Alon and Asaf Shapira. A characterization of the (natural) graph properties testable with one-sided error. SIAM J. Comput., 37(6):1703-1727, 2008. 2, 31, 33, 39

[10] Noga Alon and Joel H. Spencer. The probabilistic method. Wiley-Interscience Series in Discrete Mathematics and Optimization. John Wiley \& Sons Inc., Hoboken, NJ, third edition, 2008. With an appendix on the life and work of Paul Erdôs. 12, 43

[11] Michael A. Bender and Dana Ron. Testing properties of directed graphs: Acyclicity and connectivity. Random Struct. Algorithms, 20(2):184-205, March 2002. 9

[12] Manuel Blum, Michael Luby, and Ronitt Rubinfeld. Self-testing/correcting with applications to numerical problems. J. Comput. Syst. Sci., 47(3):549-595, December 1993. 1

[13] Herman Chernoff. A measure of asymptotic efficiency for tests of a hypothesis based on the sum of observations. The Annals of Mathematical Statistics, pages 493-507, 1952. 43

[14] David Conlon and Jacob Fox. Bounds for graph regularity and removal lemmas. Geom. Funct. Anal., 22(5):1191-1256, 2012. 26

[15] Reinhard Diestel. Graph theory, volume 173 of Graduate Texts in Mathematics. Springer, Heidelberg, fourth edition, 2010. 3 
[16] Eldar Fischer. The art of uninformed decisions: A primer to property testing. Science, 75:97126, 2001. 1

[17] Eldar Fischer. Testing graphs for colorability properties. Random Struct. Algorithms, 26(3):289-309, May 2005. 2, 35

[18] Eldar Fischer and Ilan Newman. Testing versus estimation of graph properties. SIAM J. Comput., 37(2):482-501, May 2007. 41, 42

[19] O. Goldreich and D. Ron. Property testing in bounded degree graphs. Algorithmica, 32(2):302343, 2002. 1,6

[20] Oded Goldreich. A brief introduction to property testing. In Oded Goldreich, editor, Property Testing, pages 1-5. Springer-Verlag, Berlin, Heidelberg, 2010. 1

[21] Oded Goldreich, Shafi Goldwasser, and Dana Ron. Property testing and its connection to learning and approximation. J. ACM, 45(4):653-750, 1998. 1, 6

[22] Oded Goldreich and Luca Trevisan. Three theorems regarding testing graph properties. Random Structures Algorithms, 23(1):23-57, 2003. 8

[23] Oded Goldreich and Luca Trevisan. Errata to the paper: Three theorems regarding testing graph properties. http://www.wisdom.weizmann.ac.il/〜oded/PS/tt-err.ps, 2005. 8

[24] W. T. Gowers. Lower bounds of tower type for Szemerédi's uniformity lemma. Geom. Funct. Anal., 7(2):322-337, 1997. 19

[25] János Komlós, Ali Shokoufandeh, Miklós Simonovits, and Endre Szemerédi. The regularity lemma and its applications in graph theory. In Theoretical aspects of computer science (Tehran, 2000), volume 2292 of Lecture Notes in Comput. Sci., pages 84-112. Springer, Berlin, 2002. 12, 13

[26] László Lovász. Large networks and graph limits, volume 60 of American Mathematical Society Colloquium Publications. American Mathematical Society, Providence, RI, 2012. 2

[27] J.W. Moon. Topics on Tournaments. Holt, Rinehart and Winston, 1968. 4, 5

[28] Michal Parnas and Dana Ron. Testing the diameter of graphs. Random Structures Algorithms, 20(2):165-183, 2002. 6

[29] Ronitt Rubinfeld and Madhu Sudan. Robust characterizations of polynomials with applications to program testing. SIAM J. Comput., 25(2):252-271, 1996. 1

[30] Endre Szemerédi. Regular partitions of graphs. In Problèmes combinatoires et théorie des graphes (Colloq. Internat. CNRS, Univ. Orsay, Orsay, 1976), volume 260 of Colloq. Internat. CNRS, pages 399-401. CNRS, Paris, 1978. 12, 18, 26

gr indica que o resultado referenciado é na verdade uma versão análoga (do resultado em questão) para o caso de grafos. 